\title{
Retaining Structure Force-Deformation Analysis Model for an Ultradeep Foundation Pit
}

\author{
Qi Hu \\ College of Civil Engineering and Architecture, Zhejiang University of Technology, Hangzhou 310014, China \\ Correspondence should be addressed to Qi Hu; huqi@zju.edu.cn
}

Received 5 April 2013; Revised 17 September 2013; Accepted 3 October 2013

Academic Editor: Asier Ibeas

Copyright (c) 2013 Qi Hu. This is an open access article distributed under the Creative Commons Attribution License, which permits unrestricted use, distribution, and reproduction in any medium, provided the original work is properly cited.

\begin{abstract}
An ultradeep foundation pit is a complex system composed of a retaining structure, foundation soil, and groundwater. Design and construction of foundation pits for use at greater depths than in the past require continual improvement in the design methods and analysis methods applied. In this paper, a load-deformation analysis model of a retaining structure based on a bearing-mode analysis of an ultra-deep foundation pit is proposed. A calculation method was theoretically derived for the horizontal foundation stiffness coefficient for this model, and the influences of factors such as space size, stress path, load level, and seepage were analyzed. A numerical example and a case study of an ultra-deep foundation pit in the Hangzhou Metro Line number 1 test section are presented. The calculated results for deformation of the structure and for earth pressure were found to be similar to the results obtained from elastic-plastic finite element analysis and similar to the measured results. The results of this study indicate that the proposed analysis model adequately reflects the force-deformation characteristics of an ultra-deep foundation pit and show that the proposed analysis model appropriately considers the influences of various factors.
\end{abstract}

\section{Introduction}

Numerous achievements have been reported in the study of deep foundation pits. Codes for the construction of foundation pits have been developed in numerous countries, $[1,2]$ and technical specifications have been prepared by local governments. Valuable experience in the design and construction of ultradeep foundation pits has been reported. Due to increasing depths of excavation, new support methods and construction technologies have been applied, and more stringent safety measures have been required. These advances require continuous improvement in the design methods and analysis theories for retaining structures.

The principle underlying current codes and technical specifications for the design of a foundation pit, which is a static design problem, is illustrated in Figure 1. The main steps in the design process can be summarized as follows. First, an initial state is considered in which the external earth pressure is equivalent to the active earth pressure $P_{a}$, and this state is constant. Second, part of the initial internal earth pressure is offset by the external earth pressure, and the change in the internal earth pressure is represented by the soil spring force $F_{i}=K_{i} \cdot \delta_{i}$. The influence of seepage on the water-earth pressure and the horizontal foundation stiffness coefficient is not considered. This basic design approach has been proven to be applicable to shallow foundation pit excavation.

An ultradeep foundation pit is a complex system that is composed of a retaining structure, foundation soil, and groundwater. The following characteristics of an ultradeep foundation pit are illustrated in Figure 2. (1) The water-earth pressure on a retaining structure is large, and the earth pressure on a flexible retaining structure is closely related to the deformation of the retaining structure, which cannot be described by a single model. (2) Because the unloading of the soil inside the pit is large and the stress path is complex, the influences of the stress level and stress path on soil parameters must be considered. (3) The deformation mode of a retaining structure consists of the lateral deformation of the entire pile group. The magnitude of the lateral support that the soil inside the pit provides to the retaining structure is related not only to the properties of the soil but also to the space size, that is, the excavation width $l$ and excavation depth $h$. (4) In areas with high groundwater levels, the influence of seepage on the water-earth pressure and soil parameters is significant 


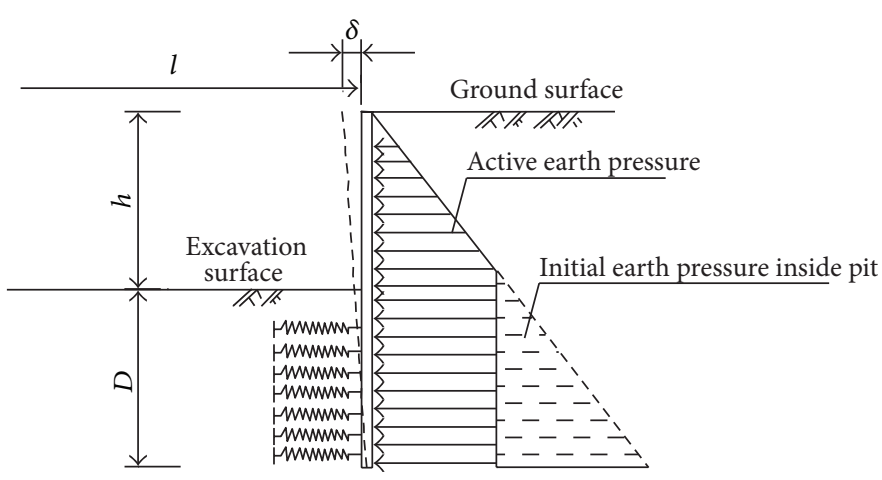

FIGURE 1: Schematic of current design approach for a foundation pit.

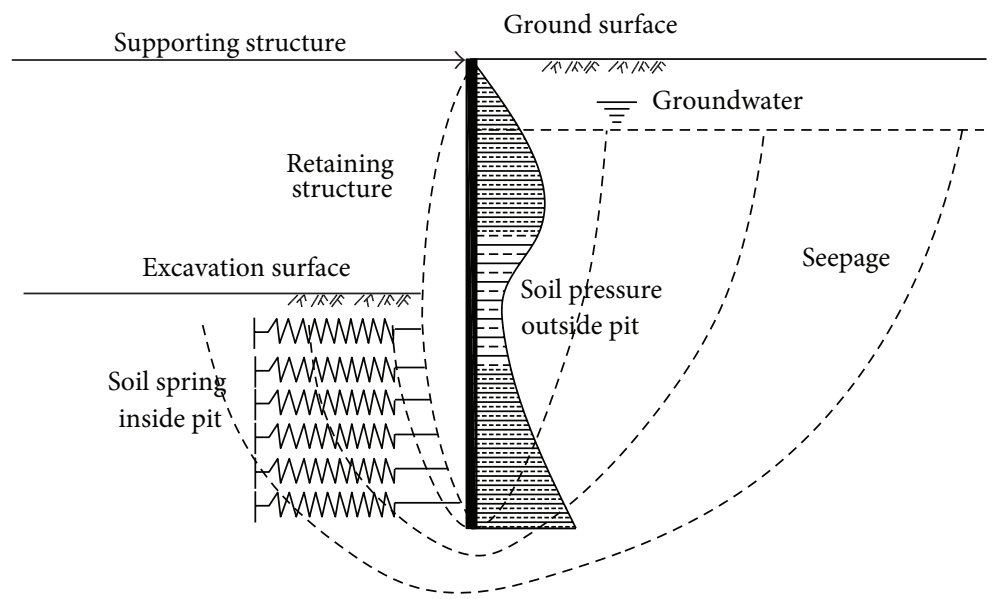

FIGURE 2: Schematic of an ultradeep foundation pit system.

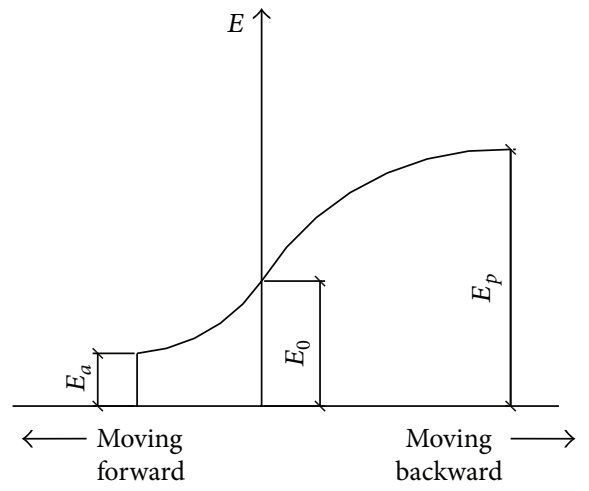

FIGURE 3: Relationship between earth pressure and deformation.

due to the large difference between the water level inside the pit and the water level outside the pit. Under the influence of seepage, the effective soil stress inside the pit is reduced and the deformation of the retaining structure is increased.

Many researchers have studied these problems. As Figure 3 shows, the displacement-dependent earth pressure theory assumes that the earth pressure consists of active earth pressure and passive earth pressure. $\mathrm{Xu}$ [3] used trigonometric functions to describe the relationship between earth pressure and deformation. Chen et al. [4] and Zhao et al. [5] used exponential functions to describe the relationship between earth pressure and deformation. Bei and Zhao [6] analyzed the relationship between active earth pressure and the deformation of a retaining structure. Many researchers, such as Lade and Duncan [7], Yuan et al. [8], Liu and Hou [9], Liu [10], and Charles and Qun [11], have conducted soil stress path experiments on foundation pits. The current methods for determining horizontal foundation stiffness coefficient values can be classified into three categories. The first category encompasses empirical methods. The value of the horizontal foundation stiffness coefficient is selected on the basis of analyses of soil geological conditions, as well as experience with similar projects and codes $[12,13]$. These methods are sometimes arbitrary. The second category encompasses field test methods, including horizontal static load tests, pressure meter tests, and flat dilatometer tests [14]. The third category encompasses laboratory test methods. According to certain theories, the relationships between the horizontal foundation stiffness coefficient $K$ and the soil modulus $E_{s}$ and shear strength $C_{u}$ can be expressed by the equations $K=\alpha E_{s}$ [15] and $K=\beta \cdot C_{u}[16]$, respectively.

Although these three types of methods have yielded useful results, none of them reflects the force-deformation behavior of an ultradeep foundation pit or considers 


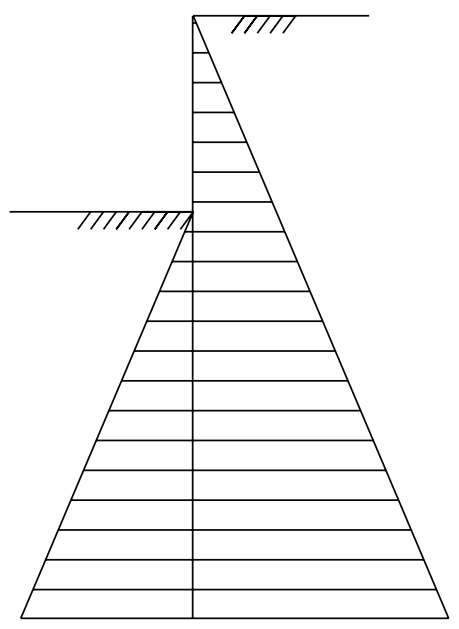

Static earth pressure

(a) Loading system

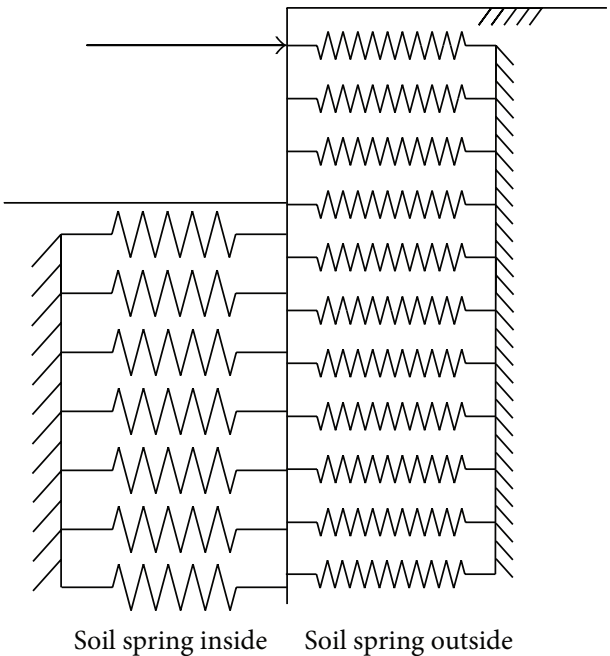

(b) Supporting system

FIGURE 4: Analysis model for an ultradeep foundation pit.

the influence of various factors on that behavior. This paper proposes an analysis model for an ultradeep foundation pit, based on the force-deformation behavior of a retaining structure and soil. A calculation method was theoretically derived for the horizontal foundation stiffness coefficient in this model. The influences of the space size, stress path, load level, and seepage on the force-deformation behavior of a retaining structure were examined. The proposed method was verified using a numerical example and a case study of an ultradeep foundation pit in the Hangzhou Metro Line number 1 test section.

\section{Analysis Model for an Ultradeep Foundation Pit}

An analysis model framework for an ultradeep foundation pit, which can be decomposed into the processes described below, is shown in Figure 4. If deformation of a retaining structure does not occur after excavation, the earth pressure outside the pit maintains a static state. Unbalanced earth pressure is sustained by the support system, which is composed of soil springs inside and outside the pit. Due to the effect of the unbalanced earth pressure between the inside and the outside of the pit, the soil springs inside the pit are compressed, the earth pressure inside the pit increases, the soil springs outside the pit are stretched, and the earth pressure outside the pit decreases, until a new balanced state is achieved and formed. Three significant differences between this model and existing analysis models exist. (1) The initial loading state consists of static earth pressure without active earth pressure. (2) The change in earth pressure outside the pit is considered using the force of the soil springs outside the pit. (3) The soil spring stiffness or horizontal foundation stiffness coefficient $K$ is related not only to the soil properties but also to the loading modes of the retaining structure and the soil.

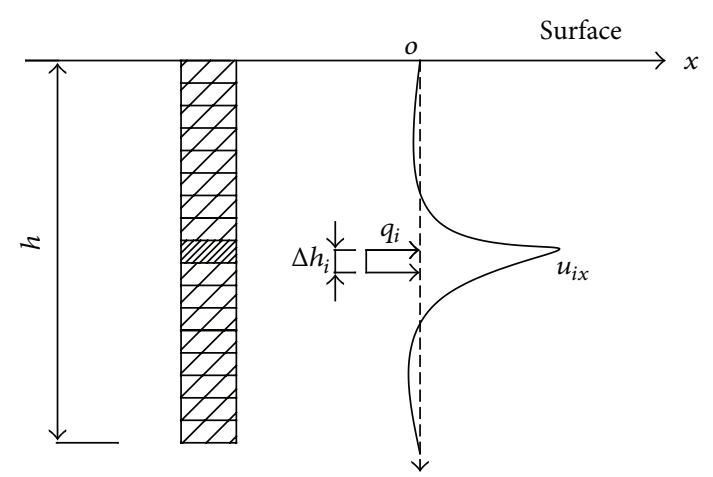

FIGURE 5: Illustration of the concept of the horizontal foundation stiffness coefficient.

As Figure 5 shows, according to the definition of a Winkler elastic foundation, the force on a soil spring is defined by $q_{i} \cdot w \cdot \Delta h$, and the soil spring stiffness is defined by $K_{i}$. $w \cdot \Delta h$. The relationships among the force, the stiffness, and the horizontal displacement of the soil spring are described by the following equations:

$$
\frac{\left(q_{i} \cdot w \cdot \Delta h\right)}{\left(K_{i} \cdot w \cdot \Delta h\right)}=\frac{q_{i}}{K_{i}}=u_{i x} \quad \text { or } \quad K_{i}=\frac{q_{i}}{u_{i x}},
$$

where $q_{i}$ denotes the horizontal strip load (in units of pressure), $w$ denotes the calculated horizontal width, and $\Delta h$ denotes the calculated thickness.

As Figure 6 shows, the soil applies lateral pressures $P_{o}$ and $P_{i}$ to the retaining structure, and the retaining structure applies lateral pressures $P_{o}^{\prime}$ and $P_{i}^{\prime}$ to the soil. If the relationship between the change in the horizontal strip load $\Delta q_{i}$ and the horizontal displacement $u_{i x}$ is established, the value of the horizontal foundation stiffness coefficient $K_{i}=\Delta q_{i} / u_{i x}$ for any depth can be determined. 


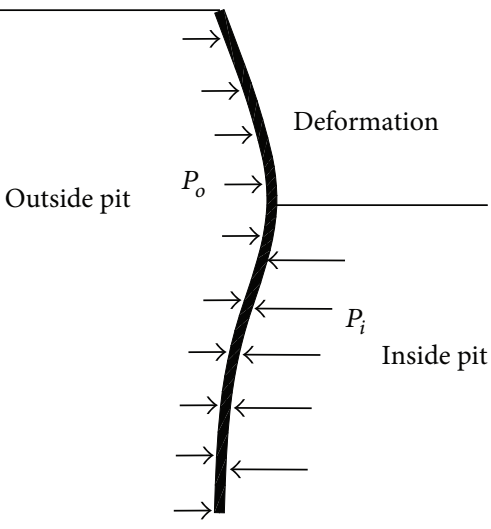

(a)

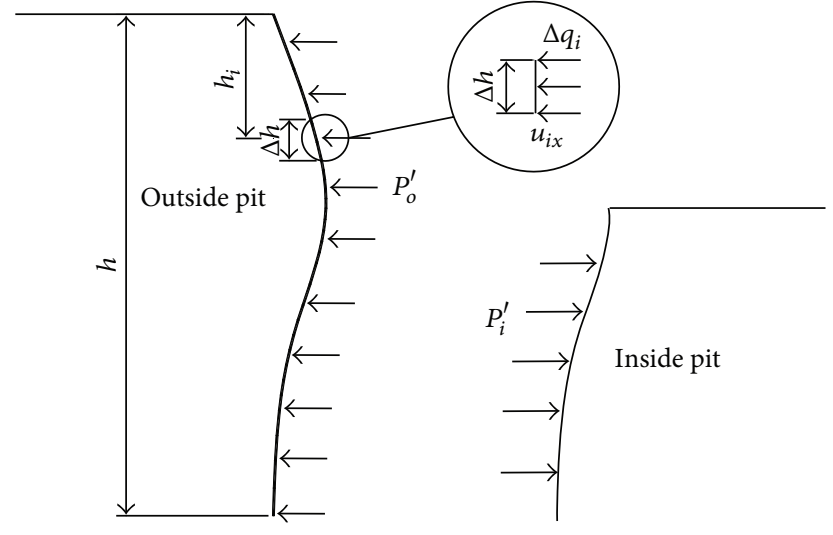

(b)

FIGURE 6: Force-deformation behavior of the retaining structure and the soil of an ultradeep foundation pit.

The solutions for the components of stress at any point in a semi-infinite elastic space due to a linear horizontal load $q$ applied at a depth $d$ (as illustrated in Figure 7) were proposed by Melan [17]. The solutions for stress at any point in a semi-infinite elastic space solution due to a uniform horizontal strip load $\bar{q}$ (as illustrated in Figure 8), as well as the displacement solution and the horizontal foundation stiffness coefficient, can be obtained by integrating Melan's solutions. These solutions for a uniform horizontal strip load reflect the actual force-deformation pattern of an ultradeep foundation pit.

As mentioned above, the solutions for the components of stress at any point in a semi-infinite elastic space due to a linear horizontal load $q$ applied at a depth $d$ (as illustrated in Figure 7) were proposed by Melan [17]:

$$
\begin{gathered}
\sigma_{x}=\frac{q x}{2 \pi(1-\mu)}\left\{\frac{x^{2}}{r_{1}^{4}}+\frac{x^{2}+8 d z+6 d^{2}}{r_{2}^{4}}+\frac{8 d z(d+z)^{2}}{r_{2}^{6}}\right. \\
\left.\quad+\frac{1-2 \mu}{2}\left[\frac{1}{r_{1}^{2}}+\frac{3}{r_{2}^{2}}-\frac{4 z(d+z)}{r_{2}^{4}}\right]\right\} \\
\sigma_{z}=\frac{q x}{2 \pi(1-\mu)}\left\{\frac{(z-d)^{2}}{r_{1}^{4}}-\frac{d^{2}-z^{2}+6 d z}{r_{2}^{4}}+\frac{8 d z x^{2}}{r_{2}^{6}}\right. \\
\left.\sigma_{x}+\sigma_{z}=\frac{q x}{2 \pi(1-\mu)} \quad-\frac{1-2 \mu}{2}\left[\frac{1}{r_{1}^{2}}-\frac{1}{r_{2}^{2}}-\frac{4 z(d+z)}{r_{2}^{4}}\right]\right\}, \\
\quad \times\left\{\frac{1}{r_{1}^{2}}+\frac{5}{r_{2}^{2}}-\frac{4\left(z^{2}+x^{2}\right)}{r_{2}^{4}}+\frac{2(1-2 \mu)}{r_{2}^{2}}\right\},
\end{gathered}
$$

where $q$ denotes the linear load, $\mu$ denotes Poisson's ratio, $d$ denotes the depth of the linear load, $x$ denotes the horizontal coordinate of a point in the semi-infinite space, and $z$ denotes the vertical coordinate of a point in the semi-infinite space.

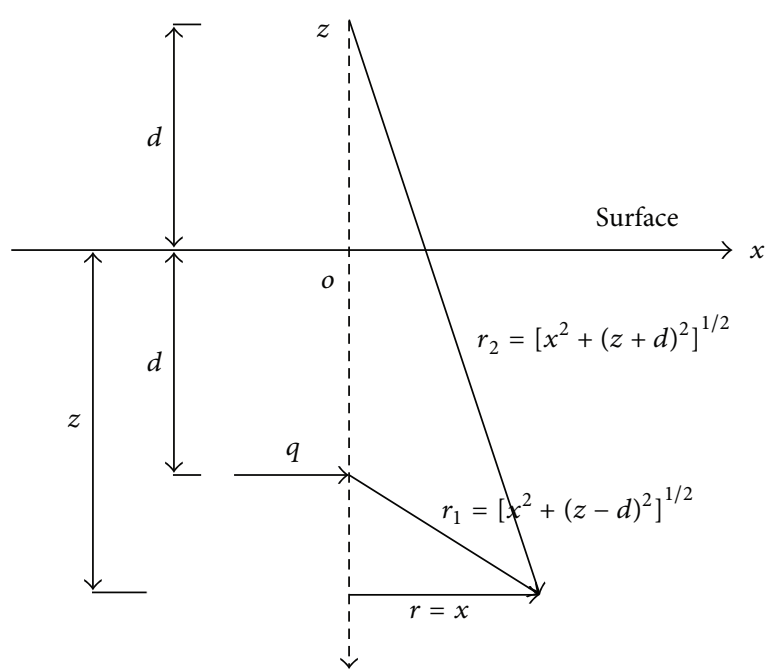

FIgURE 7: Schematic diagram of a linear horizontal load.

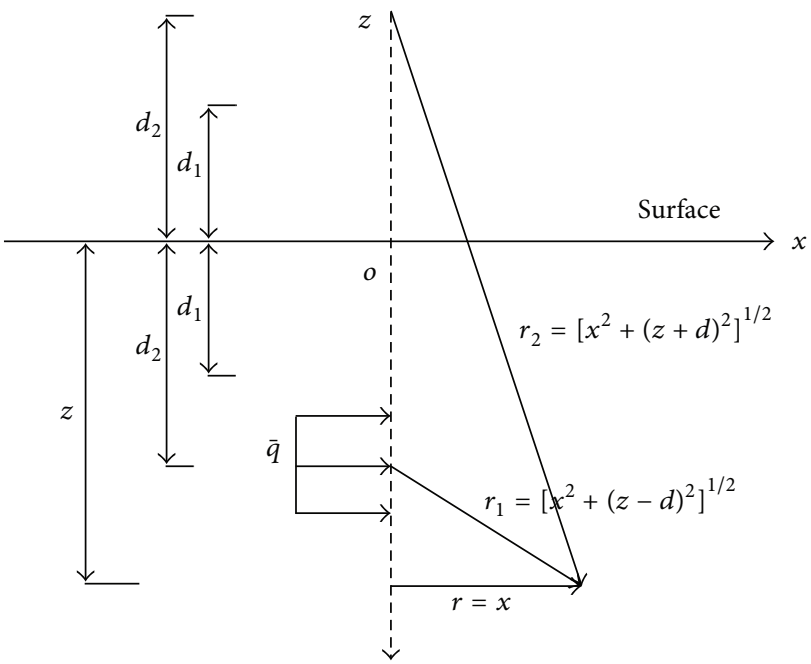

FIGURE 8: Schematic diagram of a horizontal uniform strip load. 


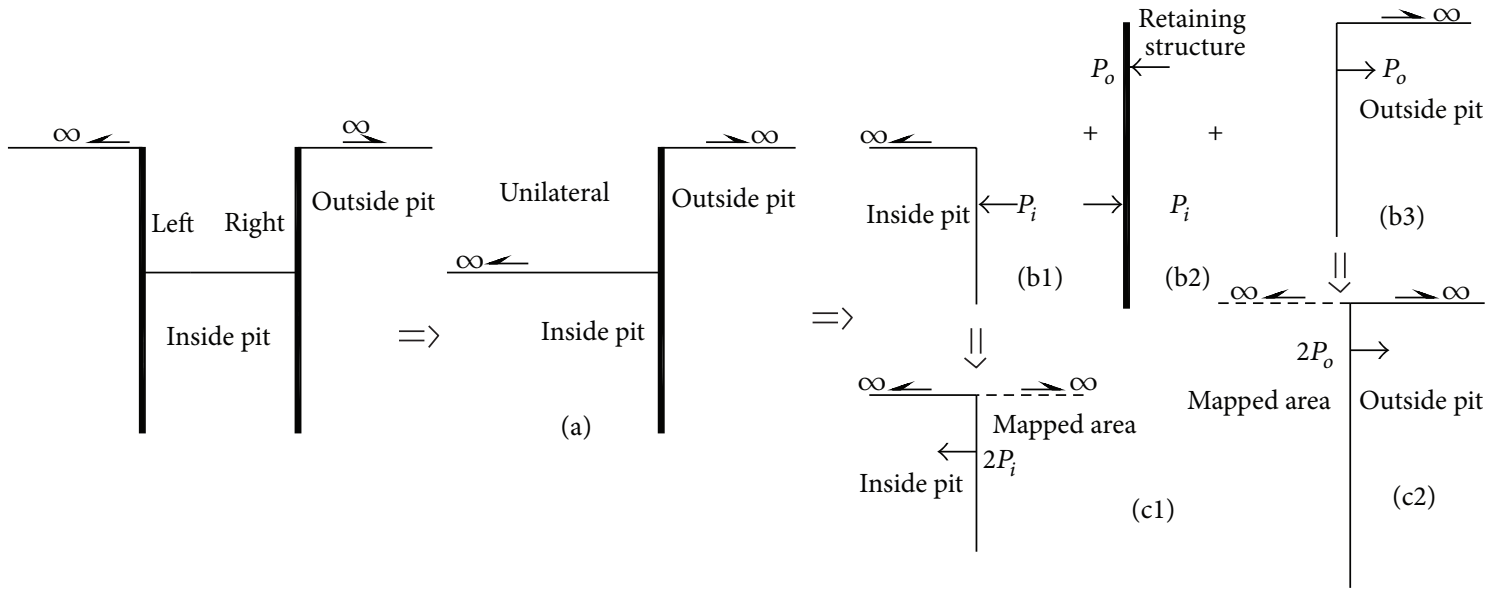

FIgURE 9: Assumption 1 for the analysis model.

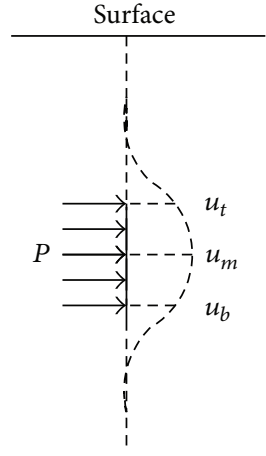

(a) Flexible load

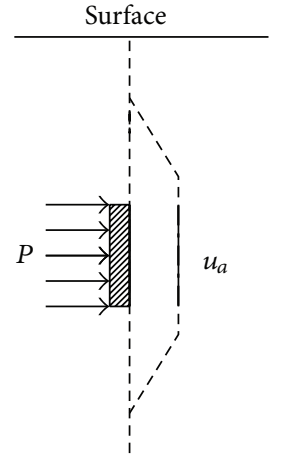

(b) Rigid base

Figure 10: Assumption 2 for the analysis model.

The solutions for the components of stress at any point in a semi-infinite elastic space due to a uniform horizontal strip load $\bar{q}$ (as illustrated in Figure 8 ) can be obtained by integrating Melan's solutions:

$$
\begin{aligned}
\sigma_{z}=\int_{d_{1}}^{d_{2}} & \frac{\bar{q} x}{2 \pi(1-\mu)} \\
& \times\left\{\frac{(z-d)^{2}}{r_{1}^{4}}-\frac{d^{2}-z^{2}+6 d z}{r_{2}^{4}}+\frac{8 d z x^{2}}{r_{2}^{6}}\right. \\
& \left.\quad-\frac{1-2 \mu}{2}\left[\frac{1}{r_{1}^{2}}-\frac{1}{r_{2}^{2}}-\frac{4 z(d+z)}{r_{2}^{4}}\right]\right\} \mathrm{d} d \\
= & \frac{\bar{q}}{2 \pi(1-\mu)} \\
& \times\left\{\frac{1}{2} \arctan \frac{d-z}{x}-\frac{x(d-z)}{2 r_{1}^{2}}\right. \\
& \quad-\frac{1}{2} \arctan \frac{d+z}{x}+\frac{2 x z d(d+z)}{r_{2}^{4}} \\
& +\frac{x(d+z)}{2 r_{2}^{2}}
\end{aligned}
$$

$$
-\frac{1-2 \mu}{2}\left(\arctan \frac{d-z}{x}\right.
$$$$
\left.\left.-\arctan \frac{d+z}{x}+\frac{2 x z}{r_{2}^{2}}\right)\right\}\left.\right|_{d=d_{1}} ^{d=d_{2}},
$$

$$
\begin{aligned}
& \sigma_{x}+\sigma_{z}=\int_{d_{1}}^{d_{2}} \frac{\bar{q} x}{2 \pi(1-\mu)}\left\{\frac{1}{r_{1}^{2}}+\frac{5}{r_{2}^{2}}-\frac{4\left(z^{2}+x^{2}\right)}{r_{2}^{4}}\right.\left.+\frac{2(1-2 \mu)}{r_{2}^{2}}\right\} \mathrm{d} d \\
&=\frac{\bar{q}}{2 \pi(1-\mu)}\left\{\arctan \frac{d-z}{x}+5 \arctan \frac{d+z}{x}\right. \\
& \quad-2\left(z^{2}+x^{2}\right) \\
& \quad \times\left(\frac{d+z}{x r_{2}^{2}}+\frac{1}{x^{2}} \arctan \frac{d+z}{x}\right) \\
&\left.+2(1-2 \mu) \arctan \frac{d+z}{x}\right\}\left.\right|_{d=d_{1}} ^{d=d_{2}}
\end{aligned}
$$




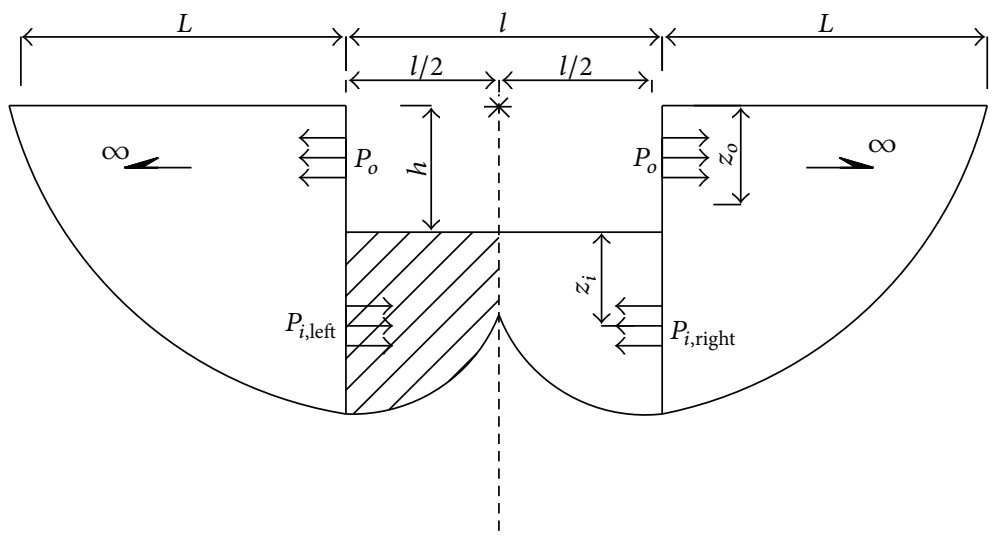

FIGURE 11: Boundary conditions for a strip foundation pit.

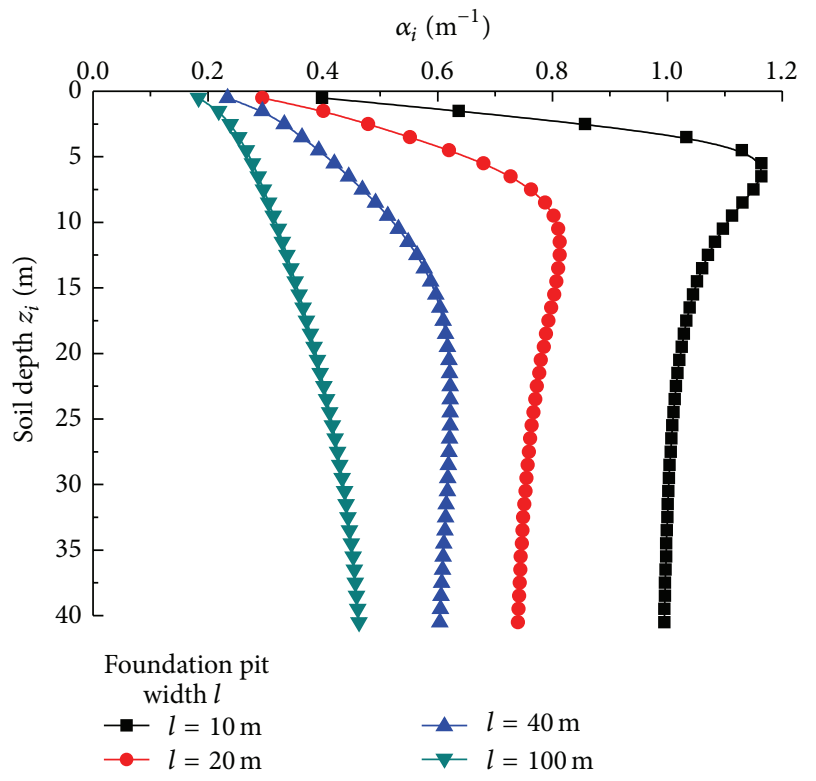

Figure 12: The coefficient $\alpha_{i}$ of the horizontal foundation stiffness coefficient inside the pit.

where $\bar{q}$ denotes the uniform strip load, $d_{1}$ denotes the depth of the top of the uniform strip load, and $d_{2}$ denotes the depth of the bottom of the uniform strip load.

2.1. Basic Assumptions of the Analysis Model. A comparison of Figures 6 and 8 reveals certain differences between the physical model of an ultradeep foundation pit and a semiinfinite space. Therefore, certain assumptions can be made.

Assumption 1. The physical model of a strip foundation pit is usually described as a plane-strain elastic problem in a semi-infinite space, as shown in Figure 9. The foundation pit is evenly divided into the left side and right side, regardless of the interaction between the left side and the right side (Figure 9(a)). Using a retaining structure as a border, the space inside and outside of the foundation pit is divided into two independent regions (Figure 9(b)). The two regions are asymmetric when subject to a lateral load and can be expanded into two separate semi-infinite elastic spaces (Figure 9(c)).

Assumption 2. Based on the provisions of settlement factors for a rigid base and a flexible load [18], if the width of the horizontal strip load is sufficiently small, the horizontal deformation of a rigid base is equivalent to the average deformation value of a flexible load, $u_{a}=\left(u_{t}+2 u_{m}+u_{b}\right) / 4$, as shown in Figure 10.

Assumption 3. For the purpose of calculating stresses, the soil is considered to be a single-phase, homogeneous, and isotropic material with a constant modulus.

2.2. Horizontal Foundation Stiffness Coefficient for the Analysis Model. According to the definition of the horizontal foundation stiffness coefficient $K=q / u_{x}$, the horizontal deformation of the isotropic plane-strain problem can be calculated from the following equations:

$$
\begin{gathered}
u_{x}=\int \varepsilon_{x} \mathrm{~d} x \\
\varepsilon_{x}=\frac{1-\mu^{2}}{E_{s}}\left(\sigma_{x}-\frac{\mu}{1-\mu} \sigma_{z}\right) \text { or } \\
u_{x}=\frac{1-\mu^{2}}{E_{s}}\left(\int \sigma_{x} \mathrm{~d} x-\frac{\mu}{1-\mu} \int \sigma_{z} \mathrm{~d} x\right) .
\end{gathered}
$$

Using the integrals of the stress solutions in (3), the displacement in semi-infinite space due to a uniform horizontal strip load $\bar{q}$ can be determined as follows:

$$
\begin{aligned}
\int \sigma_{z} \mathrm{~d} x= & \frac{\bar{q}}{2 \pi(1-\mu)} \\
\times & \left\{-\frac{z d(d+z)}{r_{2}^{2}}+\mu x \arctan \frac{d-z}{x}\right. \\
& \quad-\mu x \arctan \frac{d+z}{x}+\frac{(1-2 \mu)(z-d)}{4}
\end{aligned}
$$




$$
\begin{aligned}
& \times \ln \left(\frac{r_{1}}{d-z}\right)^{2}+\frac{(1-2 \mu)(d-z)}{4} \\
&\left.\times \ln \left(\frac{r_{2}}{d+z}\right)^{2}\right\}\left.\right|_{d=d_{1}} ^{d=d_{2}}, \\
& \int \sigma_{x} \mathrm{~d} x=\frac{\bar{q}}{2 \pi(1-\mu)} \\
& \times\left\{\frac{z d(d+z)}{r_{2}^{2}}+(1-\mu) x \arctan \frac{d-z}{x}\right. \\
&+\left(5 x-3 \mu x+\frac{2 z^{2}}{x}\right) \arctan \frac{d+z}{x} \\
&+\frac{(3-2 \mu)(d-z)}{4} \ln \left(\frac{r_{1}}{d-z}\right)^{2} \\
&+\frac{d(5-6 \mu)+z(7-10 \mu)}{4} \\
&\left.\quad \times \ln \left(\frac{r_{2}}{d+z}\right)^{2}\right\}\left.\right|_{d=d_{1}} ^{d=d_{2}} .
\end{aligned}
$$

The average displacement within the loading area is expressed as follows:

$$
\bar{u}_{x}=\frac{\left(u_{x, z=d_{1}}+2 u_{x, z=\left(d_{1}+d_{2}\right) / 2}+u_{x, z=d_{2}}\right)}{4}
$$

According to Assumption 1, the real load in a quarterinfinite space is $q=\bar{q} / 2$, due to the operation of asymmetric mapping. When (4) and (5) are used to solve the horizontal deformation equation, the integral range shown in Figure 11 must be determined. In a symmetric excavation, the horizontal integral range is finite inside the pit:

$$
u_{x}=\int_{0}^{l / 2} \varepsilon_{x} d x-\int_{l / 2}^{l} \varepsilon_{x} d x
$$

The horizontal foundation coefficient $K_{i}$ of the soil springs inside the pit can be obtained from the average displacement within the loading area:

$$
K_{i}=\frac{q}{\bar{u}_{x}}=\frac{E_{s}}{1-\mu^{2}} \frac{\bar{q}}{2}\left[\overline{\left(\int_{0}^{l / 2} \sigma_{x} \mathrm{~d} x-\int_{l / 2}^{l} \sigma_{x} \mathrm{~d} x\right)-\frac{\mu}{1-\mu}\left(\int_{0}^{l / 2} \sigma_{z} \mathrm{~d} x-\int_{l / 2}^{l} \sigma_{z} \mathrm{~d} x\right)}\right]^{-1}
$$

Determination of the theoretical influence zone, which is infinite outside the pit, is similar to the problem of determining the thickness of the underlying layer below a strip foundation. Based on the provisions of compression depth in calculating the foundation settlement [18], when the additional stress decreases to $10 \%$ of the gravity stress, the depth is defined as the compression depth. The influence zone of lateral soil deformation is assumed to satisfy the calculation when the lateral stress decreases to $10 \%$ of the horizontal load. According to the results obtained using the stress solutions in (3), when the additional horizontal stress is $10 \%$ of the horizontal load $q$, the corresponding distance is approximately ten times the loading width. Thus, ten times the foundation pit depth was defined as the influence zone outside the pit:

$$
u_{x}=\int_{0}^{10 h} \varepsilon_{x} d x
$$

The horizontal foundation coefficient $K_{o}$ of the soil springs outside the pit can be obtained from the average displacement within the loading area:

$$
K_{o}=\frac{q}{\bar{u}_{x}}=\frac{E_{s}}{1-\mu^{2}} \frac{\bar{q}}{2}\left(\overline{\int_{0}^{10 h} \sigma_{x} \mathrm{~d} x-\frac{\mu}{1-\mu} \int_{0}^{10 h} \sigma_{z} \mathrm{~d} x}\right)^{-1} .
$$

We define

$$
\begin{gathered}
\alpha_{i}=\frac{1}{1-\mu^{2}} \frac{\bar{q}}{2}\left[\overline{\left(\int_{0}^{l / 2} \sigma_{x} \mathrm{~d} x-\int_{l / 2}^{l} \sigma_{x} \mathrm{~d} x\right)-\frac{\mu}{1-\mu}\left(\int_{0}^{l / 2} \sigma_{z} \mathrm{~d} x-\int_{l / 2}^{l} \sigma_{z} \mathrm{~d} x\right)}\right]^{-1}, \\
\alpha_{o}=\frac{1}{1-\mu^{2}} \frac{\bar{q}}{2}\left(\overline{\left.\int_{0}^{10 h} \sigma_{x} \mathrm{~d} x-\frac{\mu}{1-\mu} \int_{0}^{10 h} \sigma_{z} \mathrm{~d} x\right)^{-1}} .\right.
\end{gathered}
$$




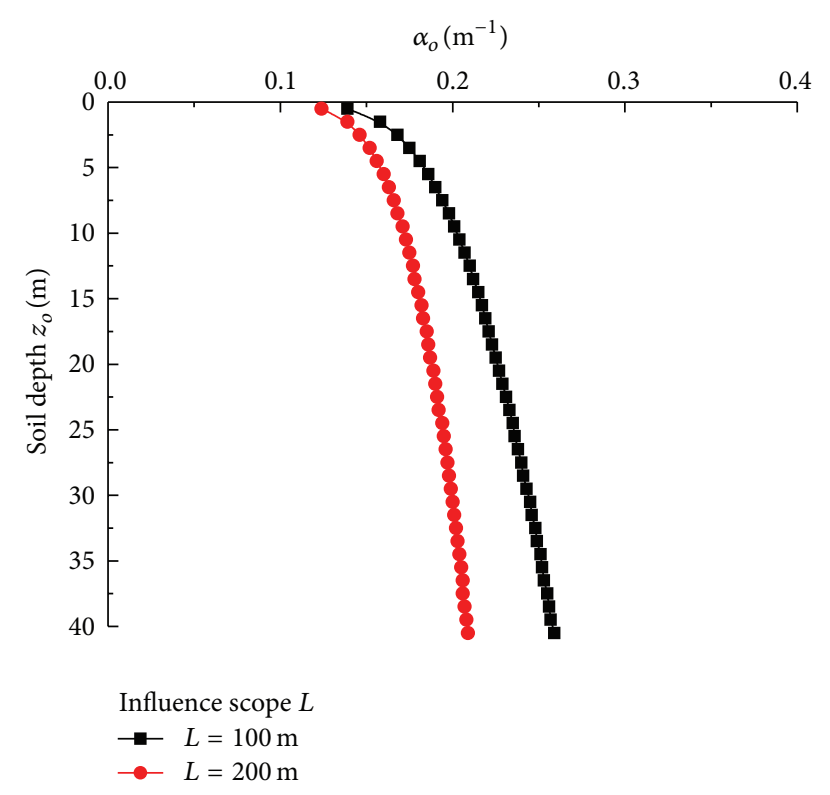

FIGURE 13: The coefficient $\alpha_{o}$ of the horizontal foundation stiffness coefficient outside the pit.

Then,

$$
\begin{aligned}
K_{i} & =\alpha_{i} E_{s}, \\
K_{o} & =\alpha_{o} E_{s},
\end{aligned}
$$

where $\alpha$ denotes the coefficient of the horizontal foundation stiffness coefficient $K$, which is related to the foundation pit space size and Poisson's ratio $\left(\mathrm{m}^{-1}\right)$, and $E_{s}$ denotes the elastic modulus of the soil.

\section{Parametric Analysis}

As shown in (8) and (10), the horizontal foundation stiffness coefficient can be described as $K=\alpha E_{s}$. The factors that influence $K$ include the space size of the foundation pit (a function of the pit width, the pit depth, and the influence zone outside the pit) and the soil parameters (elastic modulus and Poisson's ratio).

3.1. Influence of Space Size of the Foundation Pit. The coefficient $\alpha_{i}$ of the horizontal foundation stiffness coefficient for various foundation pit widths and depths is shown in Figures 12 and 13 for a Poisson's ratio of soil of 0.3 .

As Figures 12 and 13 show, the value of the coefficient $\alpha$ decreases when the foundation pit width $l$ or the influence zone $L$ increases. Due to the low level of restraint on the surface soil, the value of the coefficient $\alpha$ is also small. When the soil depth $z_{i}$ is half of the foundation pit width $l$, the coefficient $\alpha$ approaches a constant value.

3.2. Influence of Poisson's Ratio. As shown in Figure 14, the coefficient $\alpha_{i}$ decreases when Poisson's ratio $\mu$ increases. When the foundation pit is 20 meters wide, $\alpha_{i, \mu=0.1} \approx$ $1.1 \alpha_{i, \mu=0.3}$ and $\alpha_{i, \mu=0.5} \approx 0.9 \alpha_{i, \mu=0.3}$.

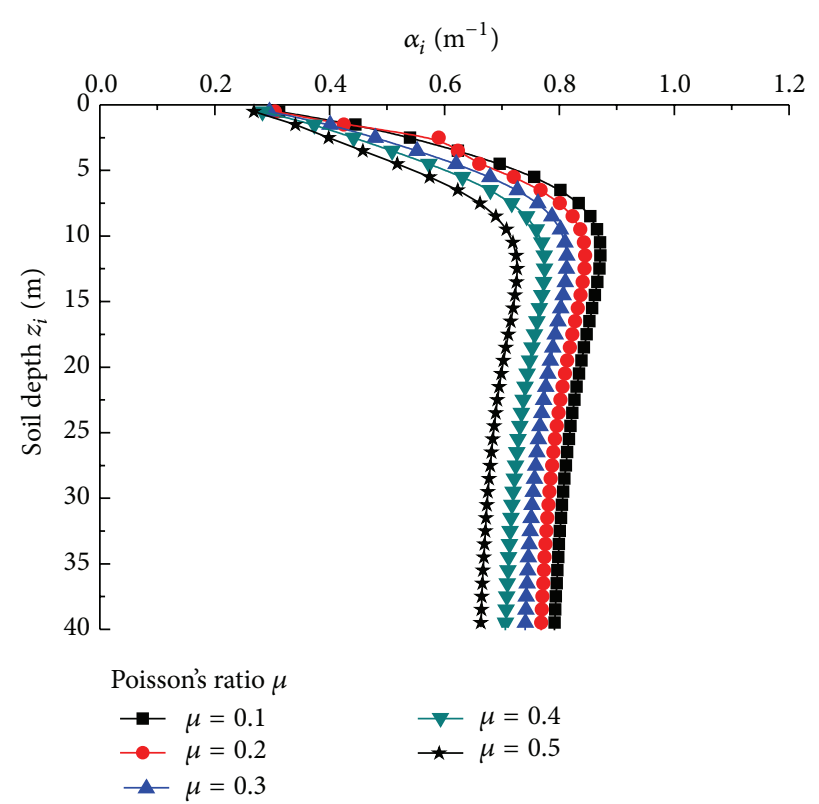

FIGURE 14: Influence of Poisson's ratio on the coefficient $\alpha_{i}$ (foundation pit width $l=20 \mathrm{~m}$ ).

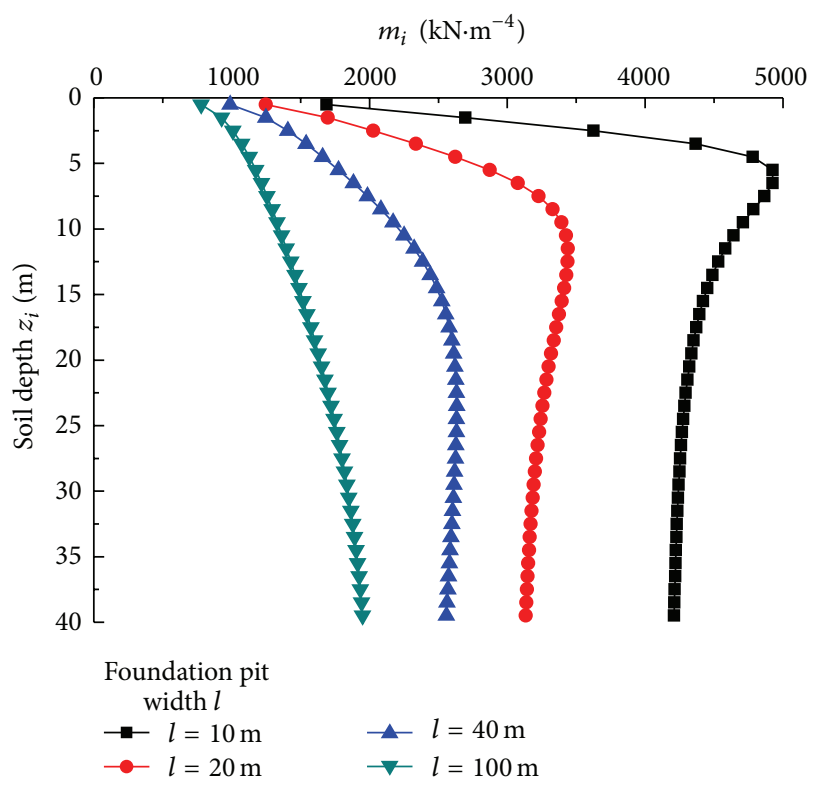

FIGURE 15: Proportional coefficient $m_{i}$ of sandy silt inside the pit.

3.3. Influence of Stress Path. The relationship between the soil modulus, the stress path, and the consolidation pressure is [19]

$$
E_{s}=\lambda \cdot \sigma^{\prime}=\lambda \cdot \gamma^{\prime} \cdot z
$$

where the coefficient $\lambda$ denotes the influence of the stress path; $\gamma^{\prime}$ denotes the soil effective gravity; and $z$ denotes the soil depth.

For the soil outside the pit, the lateral modulus coefficient $\lambda$ means the lateral unloading stress path. For the soil inside 
TABLE 1: Stress path coefficient $\lambda$ of initial tangent modulus (Wei, 2006 [20]; Hu, 2008 [19]).

\begin{tabular}{lccc}
\hline Soil layer & Stress path & $\begin{array}{c}\text { Initial tangent } \\
\text { modulus } E_{i}\end{array}$ & $\begin{array}{c}\text { Stress path } \\
\text { coefficient } \lambda\end{array}$ \\
\hline $2-3$ & $\begin{array}{c}\text { Vertical } \\
\text { compression } \\
\text { Lateral } \\
\text { unloading } \\
\text { Vertical } \\
\text { unloading } \\
\text { Vertical } \\
\text { modulus }\end{array}$ & $\begin{array}{c}\text { Vertical tangent } \\
\text { moderal tangent } \\
\text { modulus }\end{array}$ & 158 \\
$2-3$ & & 469 \\
\hline
\end{tabular}

the pit, the lateral modulus coefficient $\lambda$ means the vertical unloading stress path.

Taking the influence of the stress path into consideration, the horizontal foundation coefficient can be expressed as follows:

$$
K=\alpha \cdot E_{s}=\alpha \cdot \lambda \cdot \gamma^{\prime} \cdot z=m \cdot z .
$$

The proportional coefficient $m$ of the horizontal foundation stiffness coefficient can be expressed as $\alpha \cdot \lambda \cdot \gamma^{\prime}$. The influence of the stress path on the coefficient $\lambda$ of the initial tangent modulus $E_{i}$, according to stress path tests on Hangzhou sandy silt, is indicated in the results shown in Table 1.

The average effective gravity $\gamma^{\prime}$ of Hangzhou sandy silt is $9.0 \mathrm{kN} / \mathrm{m}^{3}$ [19]. According to the test results shown in Table 1, the lateral unloading stress path coefficient $\lambda$ is approximately 80 , and the vertical unloading stress path coefficient $\lambda$ is approximately 470 . The results for the proportional coefficient $m$ of sandy silt inside and outside the foundation pit, when Poisson's ratio $\mu$ is 0.3 , are shown in Figures 15 and 16 and Table 2.

As the values in Table 2 show, the variation in the proportional coefficient $m$ in the homogeneous foundation is similar to that of coefficient $\alpha$. The greater the width of the foundation pit is, the smaller the proportional coefficient $m$ is. The value of the proportional coefficient $m$ is smallest at the ground surface. When the soil depth is half of the foundation pit width, the proportional coefficient $m$ approaches a constant value. In general, the proportional coefficient $m$ of the soil inside the pit is considerably larger than the proportional coefficient $m$ of the soil outside the pit.

3.4. Influence of Load Level. The stress-strain behavior of soil is nonlinear. As the load level increases, the rate of strain, the soil modulus, and the horizontal foundation stiffness coefficient decrease. Thus, the effect of load level should be considered. The secant modulus for the Duncan and Chang [21] hyperbolic equations, shown in Figure 17, can be expressed as follows:

$$
E_{q}=E_{i} \cdot\left(1-\frac{\sigma_{1}-\sigma_{3}}{\left(\sigma_{1}-\sigma_{3}\right)_{\mathrm{ult}}}\right)=E_{i} \cdot\left(1-\left(\sigma_{1}-\sigma_{3}\right) \cdot b\right),
$$

where $b=\left(\sigma_{1}-\sigma_{3}\right)_{\mathrm{ult}}^{-1}$.

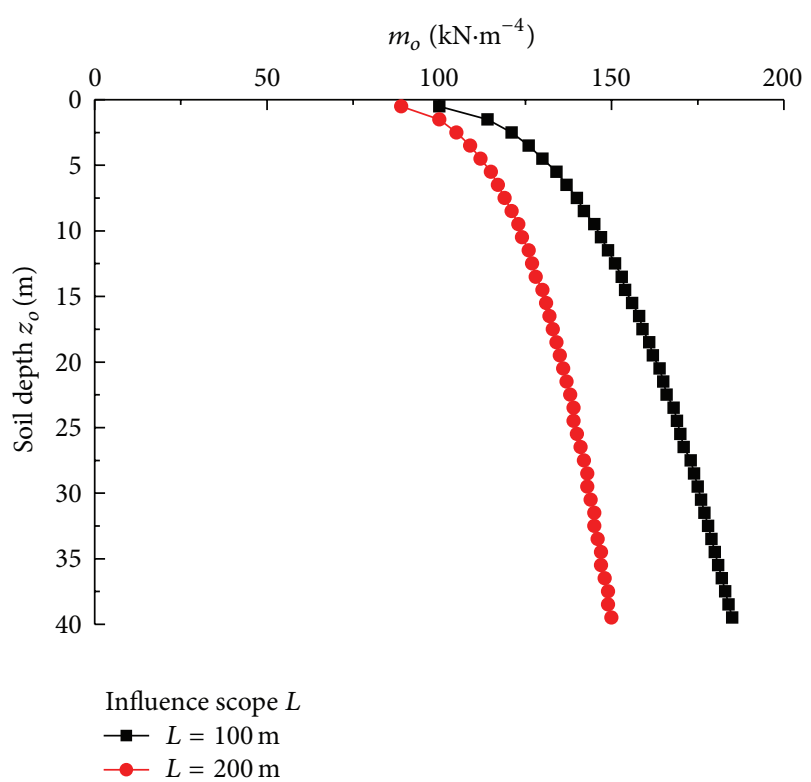

FIGURE 16: Proportional coefficient $m_{o}$ of sandy silt outside the pit.

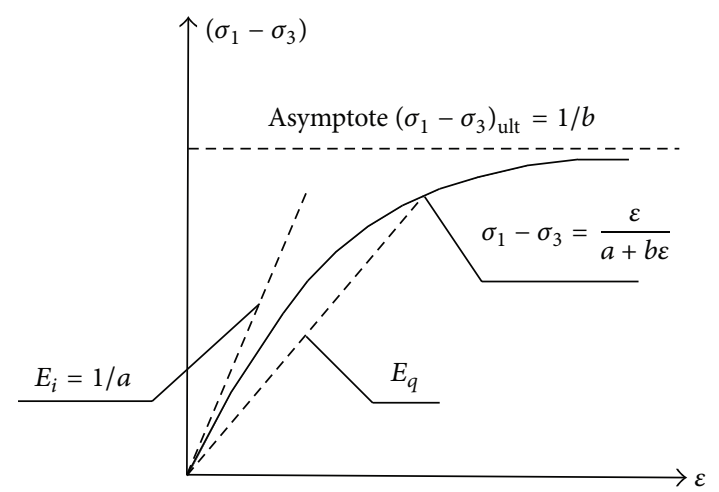

FIGURE 17: Nonlinear stress-strain relationship according to the Duncan-Chang model.

Taking the influence of load level into consideration, the horizontal foundation stiffness coefficient can be expressed as follows:

$$
\begin{aligned}
K= & \frac{q}{\bar{u}_{x}}=\frac{q}{\left(\overline{\int \varepsilon_{x} \mathrm{~d} x}\right)} \\
\varepsilon_{x} & =\frac{1-\mu^{2}}{E_{q}}\left(\sigma_{x}-\frac{\mu}{1-\mu} \sigma_{z}\right) \\
& =\frac{1-\mu^{2}}{E_{i} \cdot\left[1-\left(\sigma_{x}-\sigma_{z}\right) \cdot b\right]}\left(\sigma_{x}-\frac{\mu}{1-\mu} \sigma_{z}\right) .
\end{aligned}
$$

As in the linear elastic model, the initial tangent modulus in (16) is unrelated to the stress levels $\sigma_{x}$ and $\sigma_{z}$, and the effect of load level is only related to the coefficient $\alpha$. Equation (16) is relatively complicated and must be solved by numerical integration. The influence of the load level $q$ and the strength parameter $b$ on the coefficient $\alpha$ when the foundation pit 
TABle 2: Proportional coefficient $m$ of Hangzhou sandy silt.

\begin{tabular}{lcccccccc}
\hline \multirow{2}{*}{ Location } & \multicolumn{3}{c}{ Soil inside pit } & \multicolumn{3}{c}{ Soil outside pit } \\
& Foundation pit width L/m & 10 & 20 & 40 & 100 & Horizontal influence scope $\mathrm{L} / \mathrm{m}$ & 100 & 200 \\
\hline Ground surface & \multirow{2}{*}{$m_{i} / \mathrm{kN} \mathrm{m}^{-4}$} & 1,689 & 1,248 & 990 & 778 & & $m_{o} / \mathrm{kN} \mathrm{m}^{-4}$ & 100 \\
Deep inside & & 4,230 & 3,170 & 2,598 & 1,879 & 189 & 150 \\
\hline
\end{tabular}

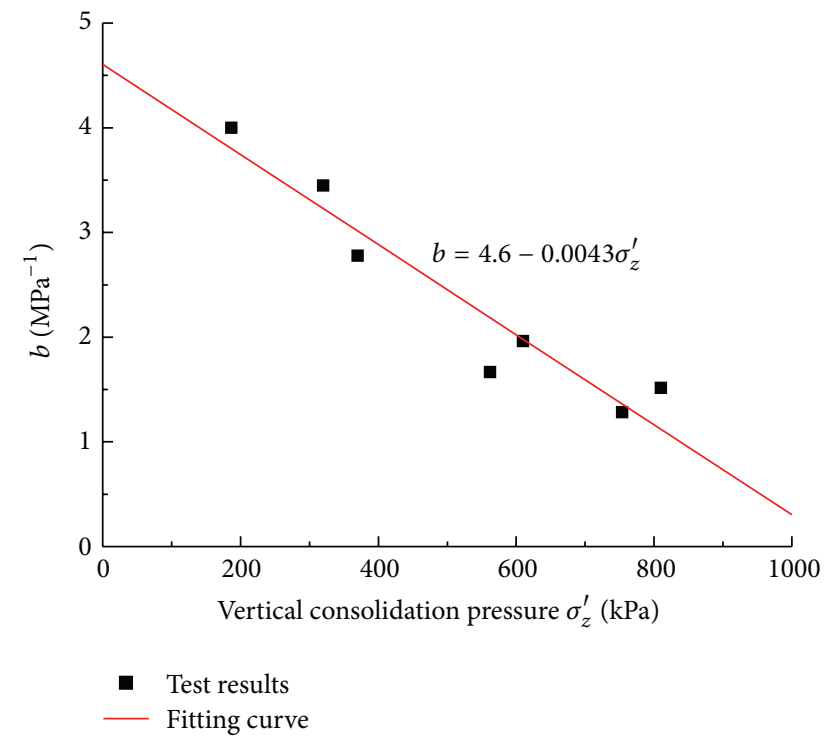

FIGURE 18: Relationship between $b$ and vertical consolidation pressure [19].

width is $20 \mathrm{~m}$ is shown in Figures 19 and 20. When the load level $q$ increases, the secant modulus of the soil and the coefficient $\alpha$ decrease. When the strength parameter $b$ increases, the soil secant modulus and the coefficient $\alpha$ decrease.

According to the stress path test results for Hangzhou sandy silt, the strength parameter $b$ in the vertical unloading stress-strain curve which is illustrated in Figure 18 can be determined from the following equation:

$$
b=4.6-0.0043 \sigma_{z}^{\prime}
$$

where $b$ has units of $\mathrm{MPa}^{-1}$ and $\sigma_{z}^{\prime}$ denotes the vertical consolidation pressure $(\mathrm{kPa})$.

3.5. Influence of Seepage. As shown in (13) and (14), the horizontal foundation stiffness coefficient and the modulus of soil are related to the soil stress state. At a site with abundant groundwater, if seepage occurs in the foundation pit, the soil stress state will change. The water-soil pressure and horizontal foundation stiffness coefficient will also be affected.

Taking the influence of seepage into consideration, the horizontal foundation stiffness coefficient of the soil inside the pit can be expressed as follows:

$$
K_{i}=\alpha_{i} \cdot E_{s}=\alpha_{i} \cdot \lambda \cdot \sigma_{z}^{\prime}=\alpha_{i} \cdot \lambda \cdot\left(\gamma_{s}^{\prime}-\gamma_{w} \cdot \bar{i}_{i}\right) \cdot z_{i}
$$

where $\bar{i}_{i}$ denotes the average hydraulic gradient inside the pit.

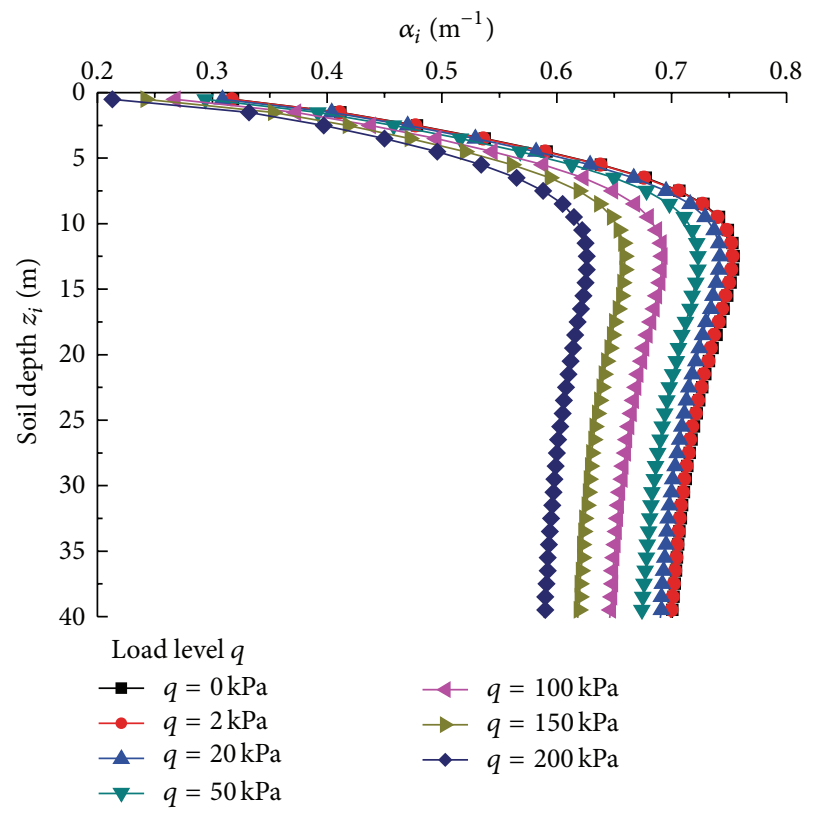

FIGURE 19: Influence of the load level $q$ on the coefficient $\alpha_{i}$ (b= $\left.3 \mathrm{MPa}^{-1}\right)$.

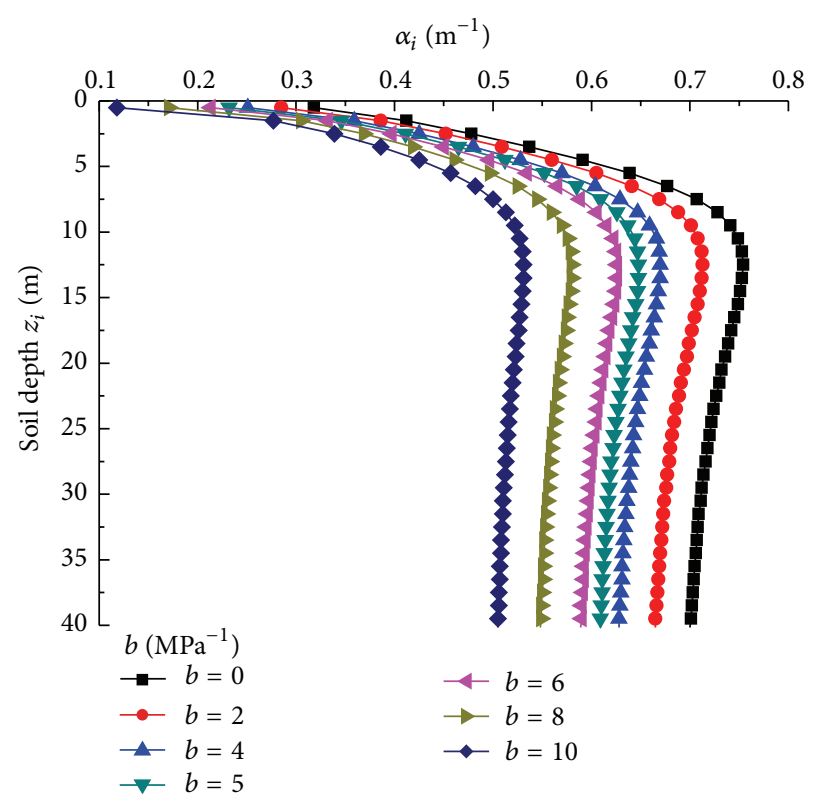

FIGURE 20: Influence of the strength parameter $b$ on the coefficient $\alpha_{i}(q=100 \mathrm{kPa})$. 


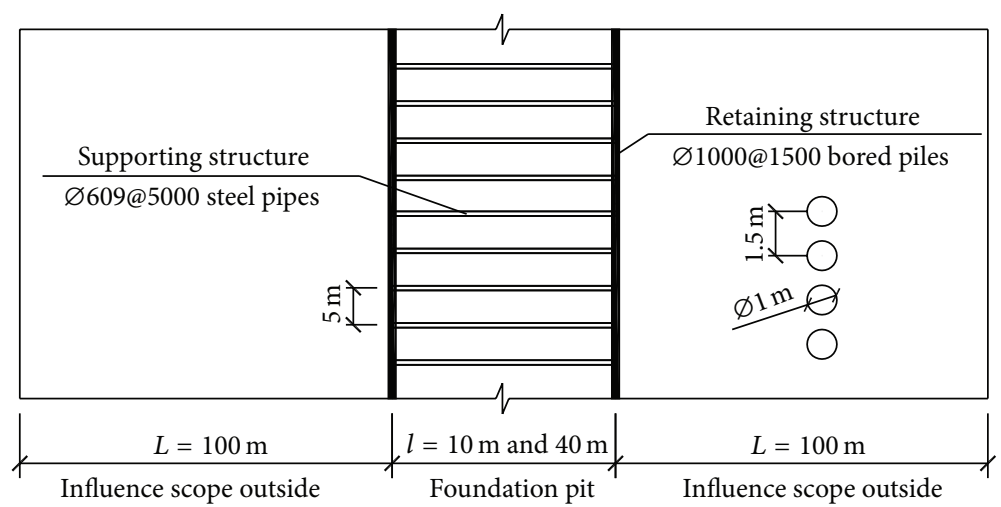

(a) Plane graph

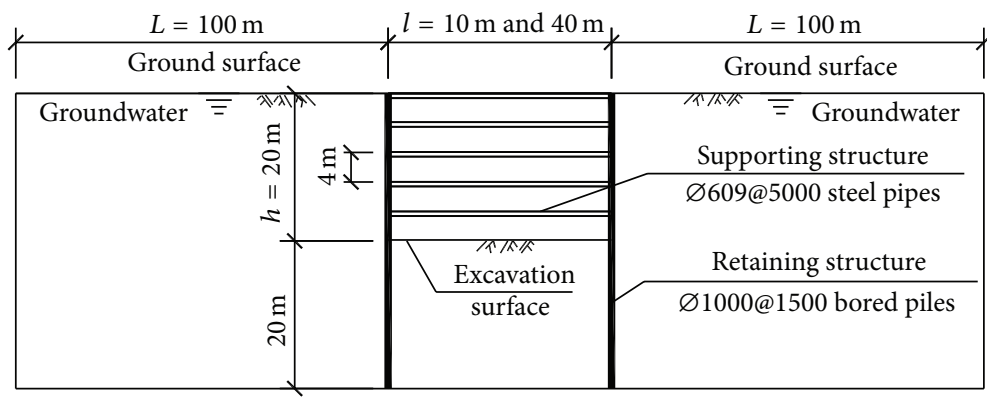

(b) Sectional graph

FIGURE 21: Foundation pit layout for the analysis example.

Taking the influence of seepage into consideration, the horizontal foundation stiffness coefficient of the soil outside the pit can be expressed as follows:

$$
K_{o}=\alpha_{o} \cdot E_{s}=\alpha_{o} \cdot \lambda \cdot \sigma_{z}^{\prime}=\alpha_{o} \cdot \lambda \cdot\left(\gamma_{s}^{\prime}+\gamma_{w} \cdot \bar{i}_{o}\right) \cdot z_{o},
$$

where $\bar{i}_{o}$ denotes the average hydraulic gradient outside the pit.

Taking the influence of seepage into consideration, the static earth pressure outside the pit, the water pressure outside the pit, and the lateral pressure outside the pit can be expressed as follows:

$$
\begin{gathered}
P_{o, s}=\left(\gamma_{s}^{\prime}+\gamma_{w} \bar{i}_{o}\right) \cdot K_{0} \cdot z_{o} \quad \text { earth pressure, } \\
P_{o, w}=\gamma_{w}\left(1-\bar{i}_{o}\right) \cdot z_{o} \quad \text { water pressure }
\end{gathered}
$$$$
P_{o}=\left(\gamma_{s}^{\prime}+\gamma_{w} \bar{i}_{o}\right) \cdot K_{0} \cdot z_{o}+\gamma_{w}\left(1-\bar{i}_{o}\right) \cdot z_{o} \quad \text { lateral pressure }
$$

where $K_{0}$ denotes the static earth pressure coefficient.

Taking the influence of seepage into consideration, the static earth pressure inside the pit, the water pressure inside

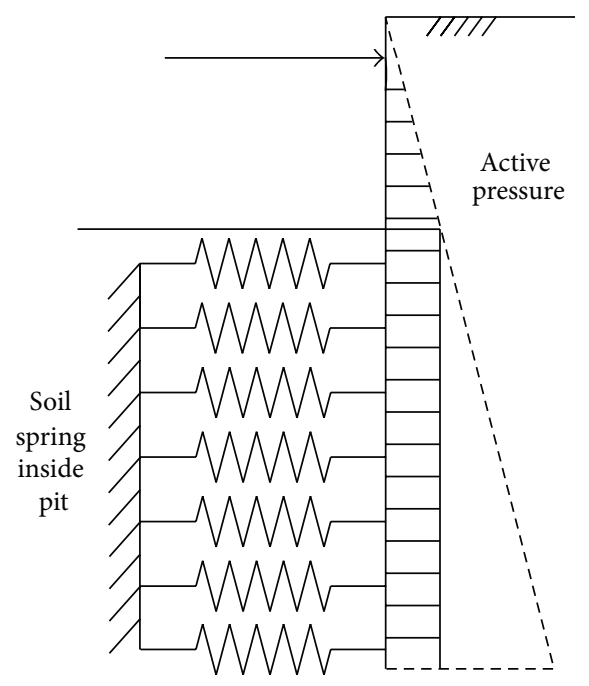

FIGURE 22: Code analysis model.

the pit, and the lateral pressure inside the pit can be expressed as follows:

$$
\begin{gathered}
P_{i, s}=\left(\gamma_{s}^{\prime}-\gamma_{w} \bar{i}_{i}\right) \cdot K_{0} \cdot z_{i} \quad \text { earth pressure, } \\
P_{i, w}=\gamma_{w}\left(1+\bar{i}_{i}\right) \cdot z_{i} \quad \text { water pressure }
\end{gathered}
$$$$
P_{i}=\left(\gamma_{s}^{\prime}-\gamma_{w} \bar{i}_{i}\right) \cdot K_{0} \cdot z_{i}+\gamma_{w}\left(1+\bar{i}_{i}\right) \cdot z_{i} \quad \text { lateral pressure. }
$$ 

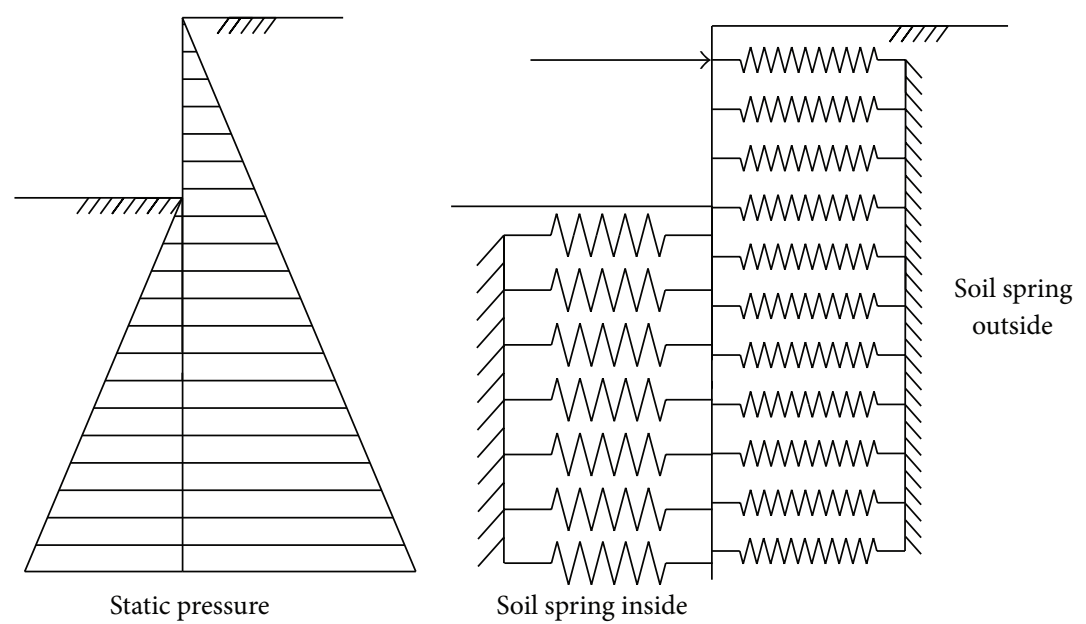

FIGURE 23: Analysis model proposed in this study.

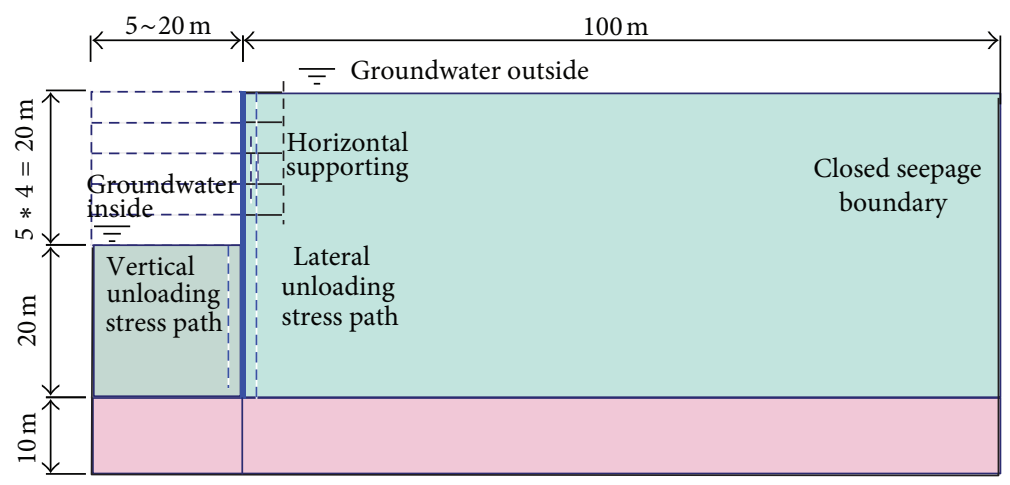

FIGURE 24: Continuum elastic-plastic-medium finite element model.

\section{Analysis Example}

The parameters for the analysis example, illustrated in Figure 21, are as follows: an excavation depth of $20 \mathrm{~m}$, five excavation steps, horizontal supporting structures consisting of 5 layers of $\varnothing 609 \times 16 @ 5000$ steel pipes, a stiffness of $E A / D / l=1400 / l \mathrm{MN} / \mathrm{m}^{2}$, retaining structures consisting of Ø1000@1500 bored piles with embedded depths of $20 \mathrm{~m}$, a concrete modulus $E_{c}=30 \mathrm{GPa}$, excavation widths of $10 \mathrm{~m}$ and $40 \mathrm{~m}$, a horizontal zone of influence of $100 \mathrm{~m}$ outside the pit, a water surface elevation equal to the ground surface elevation, and a water pressure unrelated to the earth pressure.

The foundation soil consists of sandy silt with the following characteristics: saturated gravity $\gamma_{s}=19.0 \mathrm{kN} / \mathrm{m}^{3}$, effective gravity $\gamma^{\prime}=9.0 \mathrm{kN} / \mathrm{m}^{3}$, Poisson's ratio $\mu=0.3$, shear strength parameters $c^{\prime}=5 \mathrm{kPa}$ and $\varphi^{\prime}=30^{\circ}$, lateral unloading stress path coefficient $\lambda$ of the initial tangent modulus of 80 , and vertical unloading stress path coefficient $\lambda$ of the initial tangent modulus of 470 . The calculation methods and models are shown in Figures 22, 23, and 24, and the parameters are listed in Table 3.

4.1. Analysis Results for the Retaining Structures. Figures 25 to 28 illustrate the following points. (1) The influence of the foundation pit space size and seepage cannot be considered, and the earth pressure outside the pit and the proportional coefficient $m$ of the horizontal foundation stiffness coefficient are constant. Thus, the horizontal displacement and the bending moment determined by the code method are smaller than the horizontal displacement and the bending moment determined using the other two methods. The difference increases when the excavation width increases. (2) The method proposed in this study considers the influences of space size, stress path, load level, and seepage. The results obtained using this method are similar to those obtained for the continuum elastic-plastic-medium finite element model.

Equations (18) to (21) illustrate the following points. First, due to the influence of seepage, the water pressure outside the pit $P_{o, w}$ decreased, which caused a decrease in the horizontal displacement and bending moment of the retaining structures. Second, due to the influence of seepage, the horizontal foundation stiffness coefficient inside the pit $K_{i}$ decreased, which increased the horizontal displacement and bending moment of the retaining structures. As Figures 25 and 26 show, when the width of the foundation pit is small, the hydraulic gradient $\bar{i}_{i}$ inside the pit is large. The horizontal foundation stiffness coefficient $K_{i}$ inside the pit decreases rapidly under the influence of seepage, and 
TABLE 3: Parameters according to different methods.

\begin{tabular}{|c|c|c|c|c|c|c|}
\hline Methods & Code metho & [1] & Method propo & ed in this study & $\begin{array}{r}\text { Continuum me } \\
\text { element me }\end{array}$ & $\begin{array}{l}\text { um finite } \\
\text { lod }\end{array}$ \\
\hline \multirow[b]{2}{*}{ Parameters } & $\begin{array}{l}\text { Horizontal foundation } \\
\text { stiffness coefficient }\end{array}$ & Earth pressure & $\begin{array}{c}\text { Horizontal } \\
\text { foundation } \\
\text { coefficient }\end{array}$ & Earth pressure & Soil modulus & Model \\
\hline & $\begin{array}{c}\text { Proportional coefficient } \\
\text { of horizontal foundation } \\
\text { stiffness coefficient } m= \\
4,000\end{array}$ & $\begin{array}{c}\text { Active earth } \\
\text { pressure } \\
\text { coefficient } \\
K_{a}=0.33\end{array}$ & $\begin{array}{l}\text { Considering the } \\
\text { influence of space } \\
\text { size, stress path, } \\
\text { load level, and } \\
\text { seepage }\end{array}$ & $\begin{array}{l}\text { Static earth } \\
\text { pressure coefficient } \\
\text { considering the } \\
\text { influence of } \\
\text { seepage } K_{o}=0.5\end{array}$ & $\begin{array}{c}\text { Initial tangent } \\
\text { modulus } E_{i} \\
\text { considering the } \\
\text { influence of stress } \\
\text { path }\end{array}$ & $\begin{array}{l}\text { Mohr-Coulomb } \\
\text { elastic-plastic } \\
\text { model }\end{array}$ \\
\hline
\end{tabular}

TABLE 4: Physical and mechanical parameters of the soil.

\begin{tabular}{|c|c|c|c|c|c|c|c|}
\hline \multirow{2}{*}{ Layer number } & \multirow{2}{*}{ Soil name } & \multirow{2}{*}{$\gamma_{s} / \mathrm{kN} \mathrm{m}^{-3}$} & \multirow{2}{*}{ Void ratio $e$} & \multicolumn{2}{|c|}{ Shear strength parameters } & \multicolumn{2}{|c|}{ Permeability coefficient $/{ }^{*} 10^{-4} \mathrm{~m} \mathrm{~s}^{-1}$} \\
\hline & & & & $c / \mathrm{kPa}$ & $\varphi /^{\circ}$ & $K_{V}$ & $K_{H}$ \\
\hline $2-1$ & Sandy silt & 18.9 & 0.853 & 7.6 & 28.5 & 3.39 & 2.41 \\
\hline $2-3$ & Sandy silt & 19.2 & 0.788 & 5.6 & 31.2 & 2.39 & 2.04 \\
\hline $2-4$ & Sandy silt & 19.0 & 0.858 & 6.1 & 30.8 & 2.42 & 1.82 \\
\hline $2-5$ & Sandy silt with sand & 19.3 & 0.772 & 4.7 & 31.5 & 2.65 & 3.00 \\
\hline $2-6$ & Sandy silt & 18.7 & 0.916 & 7.9 & 29.3 & & 0.13 \\
\hline $2-7$ & Sandy silt with sand & 19.3 & 0.775 & 5.5 & 31.2 & 1.20 & 2.98 \\
\hline 5 & Muddy silty clay & 18.3 & 1.067 & 19.3 & 11.7 & \multicolumn{2}{|c|}{$\left(10^{-5}\right.$ to $\left.10^{-6} \mathrm{~cm} / \mathrm{s}\right)$} \\
\hline $6-2$ & Silty clay & 19.2 & 0.866 & 50.6 & 15.7 & & \\
\hline $6-3$ & Silty clay with silt & 20.0 & 0.69 & 34.9 & 19.6 & & \\
\hline $8-1$ & Sand & 18.7 & 0.829 & 1.6 & 32.9 & & \\
\hline
\end{tabular}

the horizontal displacement and bending moment of the retaining structures increase significantly. As Figures 27 and 28 show, when the width of the foundation pit is large, the hydraulic gradient $\bar{i}_{i}$ inside the pit is similar to the hydraulic gradient $\bar{i}_{o}$ outside the pit, and the horizontal foundation stiffness coefficient $K_{i}$ inside the pit and the water pressure $P_{o, w}$ outside the pit decrease similarly under the influence of seepage. Thus, the horizontal displacement and the bending moment of the retaining structures vary slightly.

4.2. Analysis Results for Earth Pressure. In the model described in this paper, the initial state of the load consists of static earth pressure, and the earth pressure outside the pit changes when the soil springs are tensed. Figures 29, 30, 31, and 32 highlight the results obtained for earth pressure using the method proposed in this paper, which are similar to the results obtained for the continuum elastic-plastic-medium finite element model. A comparison of the results indicates that the method proposed in this paper accurately simulates the distribution patterns and the changes in earth pressure both inside and outside the pit.

\section{Case Study}

5.1. Overview. As Figure 33 shows, the parameters of the ultradeep foundation pit in the Qiutao Road station of the Hangzhou Metro Line number 1 test section are as follows: a strip foundation pit, a $20 \mathrm{~m}$ excavation width, a $16.8 \mathrm{~m}$ excavation depth, a zone of influence of $200 \mathrm{~m}$ outside the pit, 6 excavation steps, horizontal supporting structures consisting of 5 layers of $\varnothing 609 \times 16 @ 4000$ steel pipes with stiffnesses of $E A / D / l=1750 / l \mathrm{MN} / \mathrm{m}^{2}$, retaining structures consisting of $30 \mathrm{~m}$-long Ø1000@1500 bored piles, the concrete modulus $E_{c}=30 \mathrm{GPa}$, and the water surface $2 \mathrm{~m}$ below the ground surface. The water inside the pit was pumped, and the water outside the pit was not pumped. As shown in Table 4 and Figure 34, the main soil layers are composed of permeable sandy silt. The soil layer $24 \mathrm{~m}$ below the ground surface is composed of impermeable muddy silty clay. Seepage cannot occur in muddy silty clay, so the influence of seepage can be disregarded.

The average effective gravity of sandy silt is $\gamma^{\prime}=$ $9.1 \mathrm{kN} / \mathrm{m}^{3}$. According to the measured results of the stress path tests shown in Table 1 and (14), the initial tangent modulus $E_{i, i}$ for the lateral unloading soil outside the pit is $0.73 z_{i} \mathrm{MPa}$, and the initial tangent modulus $E_{i, o}$ for the lateral unloading soil outside the pit is $4.28 z_{o} \mathrm{MPa}$. The values of the proportional coefficient $m$ for the horizontal foundation stiffness coefficients, disregarding the influence of load level, are shown in Figure 35.

5.2. Application of the Calculation Method and Model. The calculation method and model, which are equivalent to the calculation methods and models used in the example analysis, are shown in Table 3 and Figures 21 through 24. The parameters are also similar to the parameters in 
Horizontal displacement $(\mathrm{mm})$

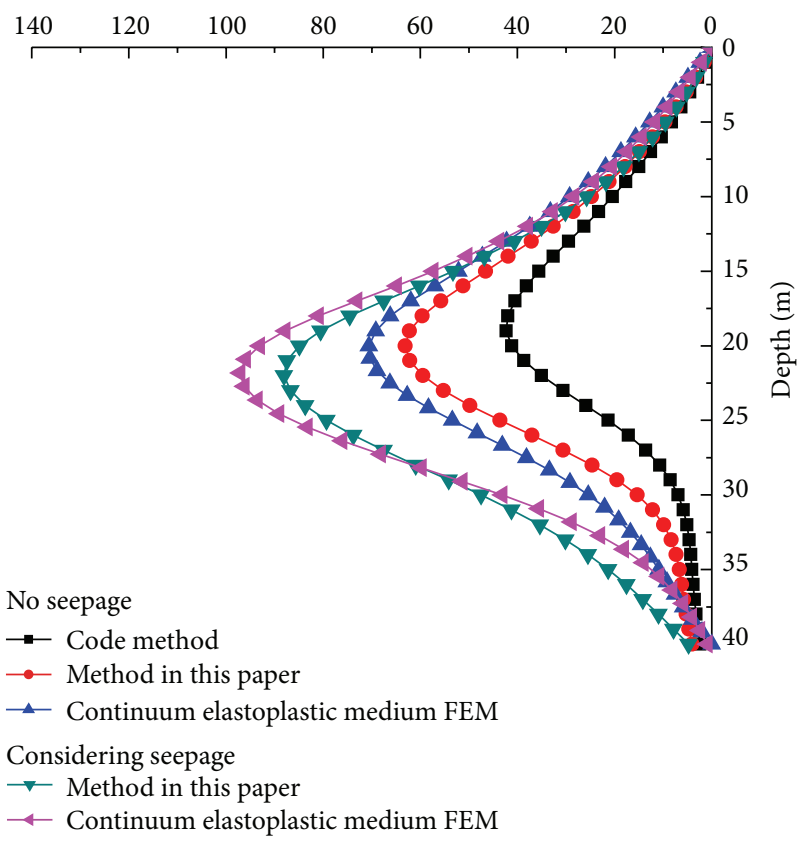

FIGURE 25: Retaining structure deformation for $10 \mathrm{~m}$ wide pit.

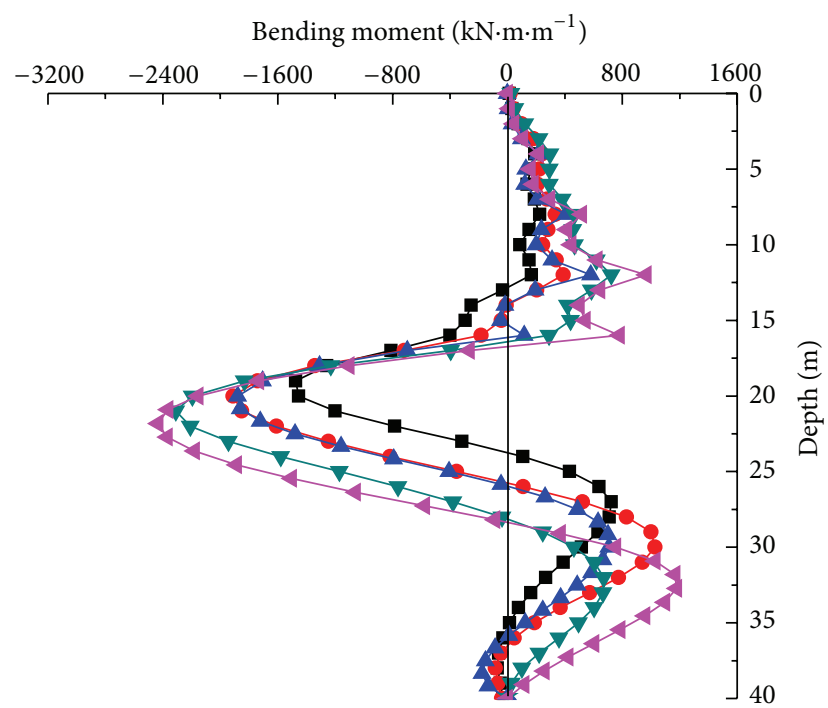

No seepage

$\rightarrow-$ Code method

$\rightarrow$ Method in this paper

— Continuum elastoplastic medium FEM

Considering seepage

$\rightarrow$ Method in this paper

$\longleftarrow$ Continuum elastoplastic medium FEM

FIGURE 26: Retaining structure bending moment for $10 \mathrm{~m}$ wide pit.

the analysis example, with the exception that the proportional coefficient $m$ in the code method is $3,000 \mathrm{kN} / \mathrm{m}^{4}$.

5.3. Analysis Results. As Figures 36 and 37 show, the results for the retaining structure deformation and earth pressure outside the pit are as follows.
Horizontal displacement $(\mathrm{mm})$

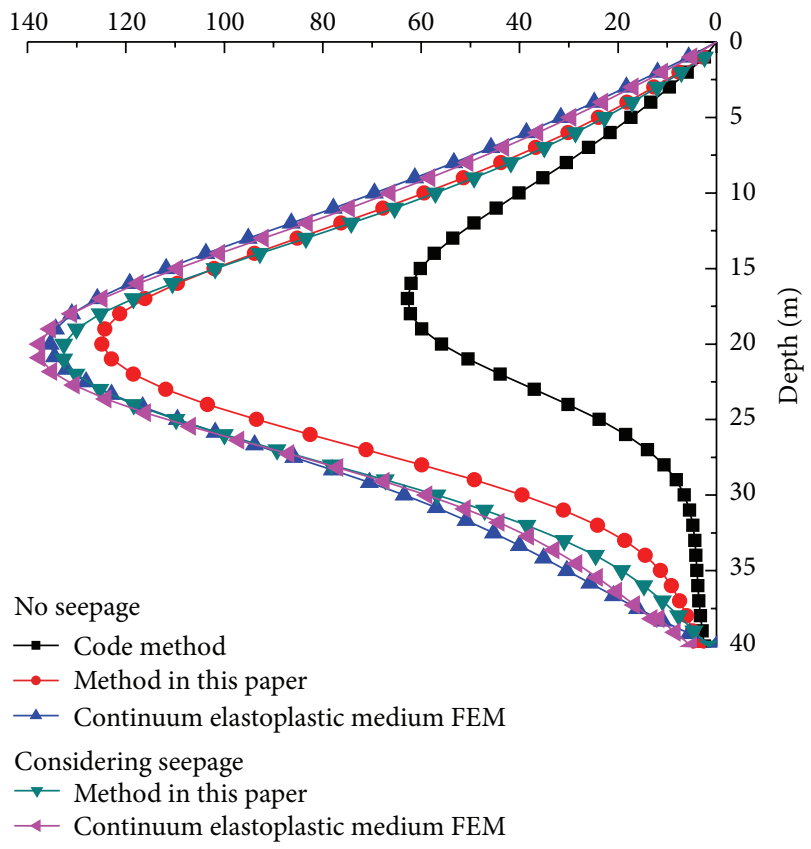

FIGURE 27: Retaining structure deformation for $40 \mathrm{~m}$ wide pit.

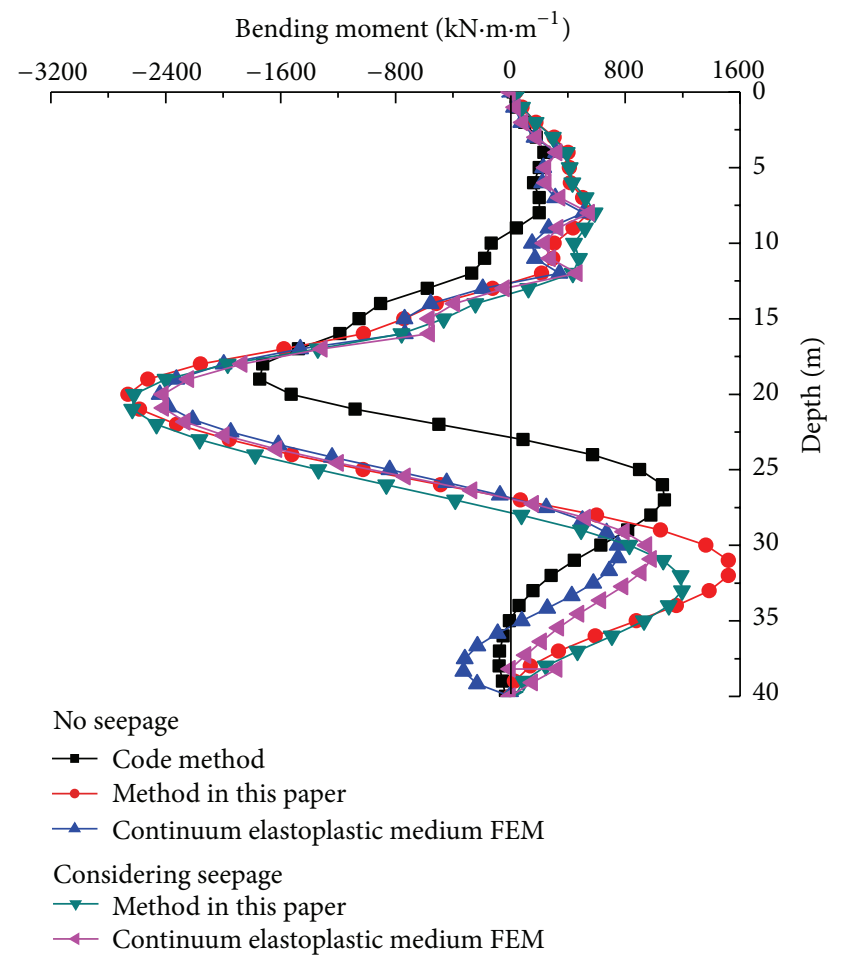

FIGURE 28: Retaining structure bending moment for $40 \mathrm{~m}$ wide pit.

(1) The results obtained using the method proposed in this paper and using the continuum elastic-plasticmedium finite element method are similar to the measured results, which indicates that the method and the model proposed in this paper can be used to accurately calculate the forces and deformations of 


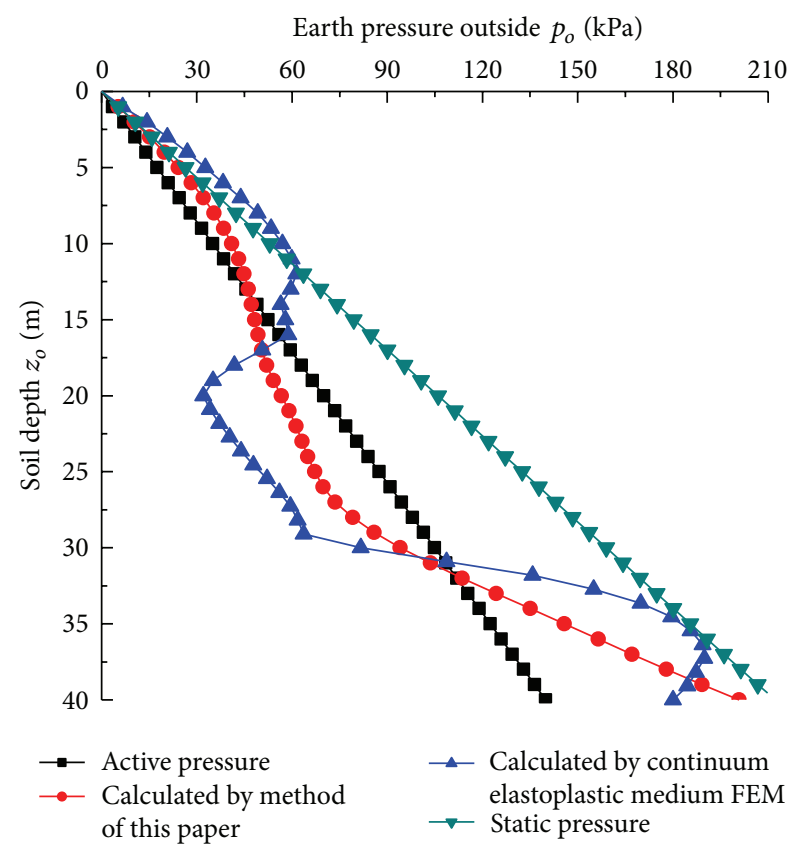

FIGURE 29: External earth pressure for $10 \mathrm{~m}$ wide pit.

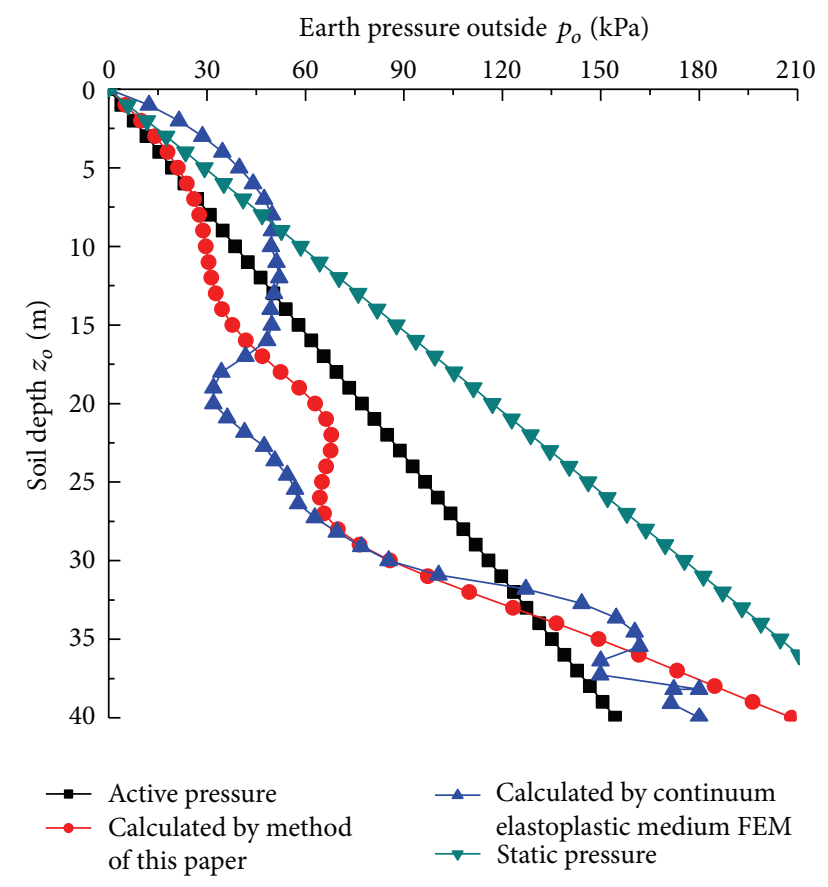

FIGURE 30: External earth pressure for $40 \mathrm{~m}$ wide pit.

the retaining structures of an ultradeep foundation pit.

(2) The influences of the stress path, the size of the foundation pit, and the stress level on the horizontal foundation stiffness coefficient are not considered in the code method. The maximum horizontal displacements calculated using the code method ranged from $32 \mathrm{~mm}$ to $35 \mathrm{~mm}$. The maximum horizontal

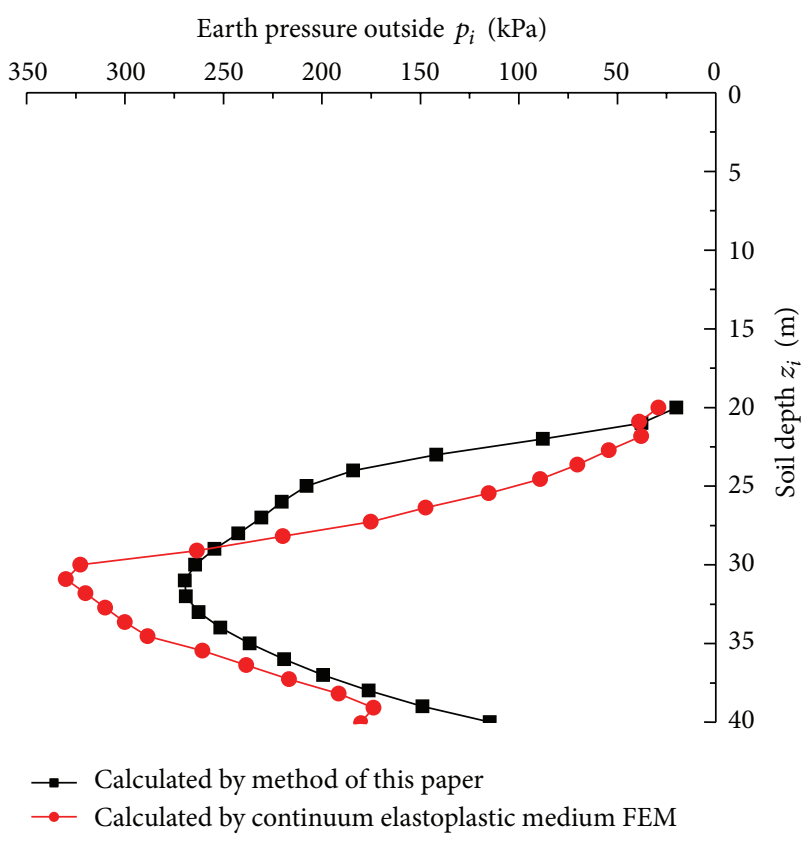

FIgURE 31: Earth pressure inside $10 \mathrm{~m}$ wide pit.

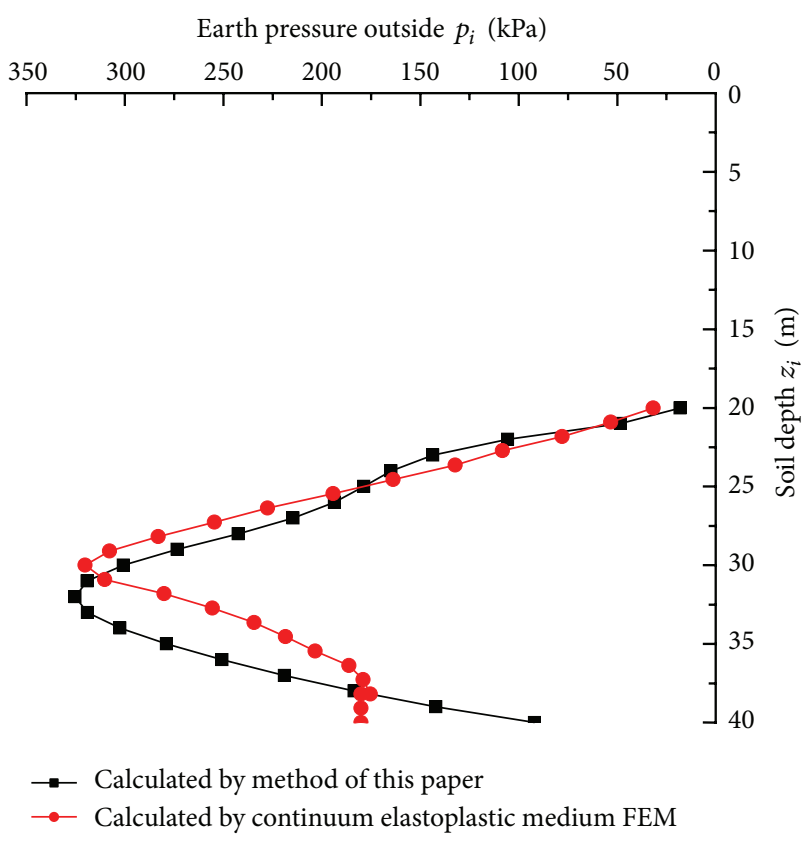

FIGURE 32: Earth pressure inside $40 \mathrm{~m}$ wide pit.

displacement calculated using the method proposed in this paper was $48 \mathrm{~mm}$, which was similar to the maximum horizontal displacement of $49 \mathrm{~mm}$ calculated using the continuum elastic-plastic-medium finite element method. The measured values ranged from $46 \mathrm{~mm}$ to $51 \mathrm{~mm}$.

(3) The results obtained for earth pressure using the method proposed in this paper were similar to 


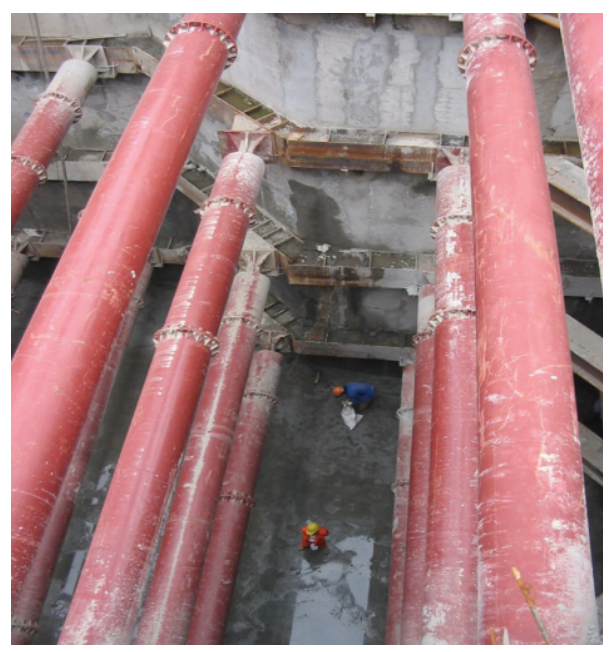

FIGURE 33: The ultradeep foundation pit in the Qiutao Road station of Hangzhou Metro Line number 1.

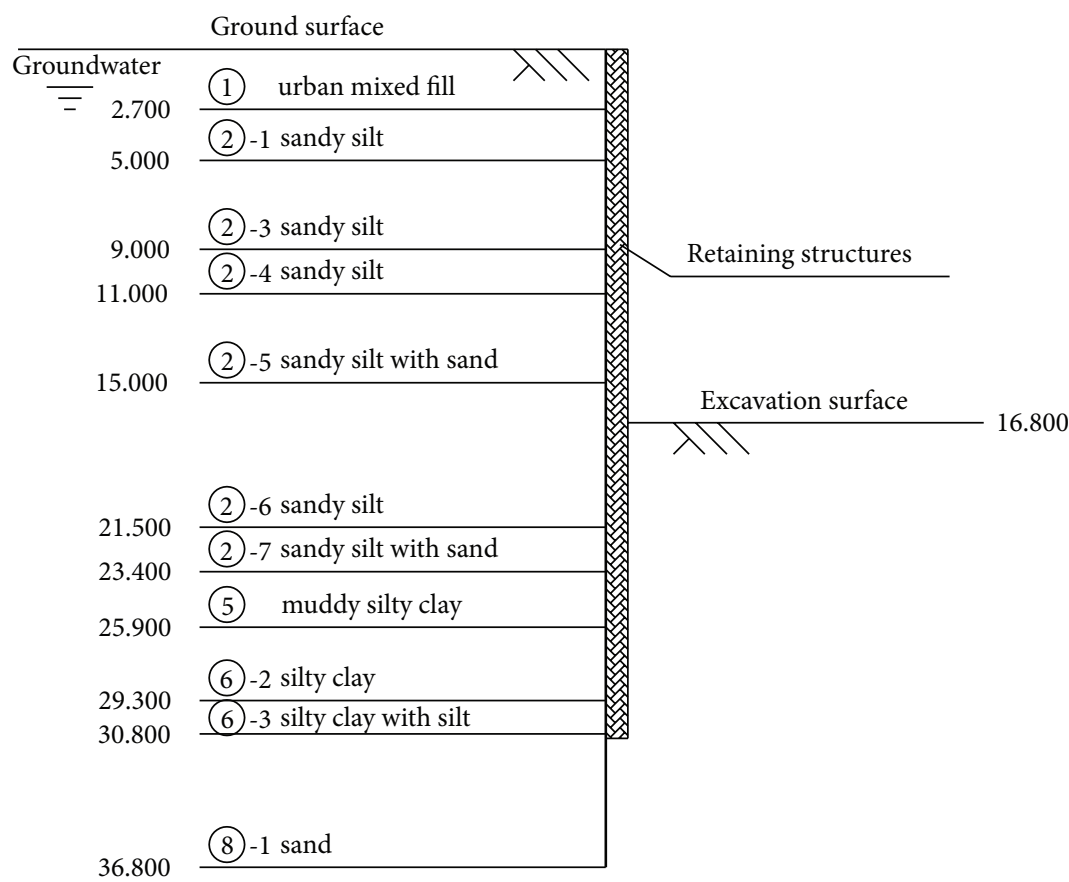

FIGURE 34: Soil profile.

the results obtained with the continuum elasticplastic-medium finite element method and were similar to the measured results.

\section{Conclusions}

New load-deformation model and method for analysis of retaining structures in ultradeep foundation pits are proposed in this paper. The horizontal foundation stiffness coefficient for this model can be expressed as $K=\alpha E_{s}$. The coefficient $\alpha$ is related to the size of the foundation pit, Poisson's ratio, the stress path and the stress level. The soil modulus $E_{s}$ is also related to the stress path, and the stress level.
(1) The value of the coefficient $\alpha$ decreases as the foundation pit width or zone of influence increases. The restraint applied to the surface of the soil is the smallest restraint; thus, coefficient $\alpha$ has the smallest value at the surface. When the soil depth is half of the depth of the foundation pit width, the coefficient $\alpha$ approaches a constant value. The value of the coefficient $\alpha$ decreases as Poisson's ratio increases.

(2) The proportional coefficient $m$ of the horizontal foundation stiffness coefficient reflects the effect of the stress path, which can be expressed as $\alpha \cdot \lambda \cdot \gamma^{\prime}$. The proportional coefficient $m$ at the ground surface 


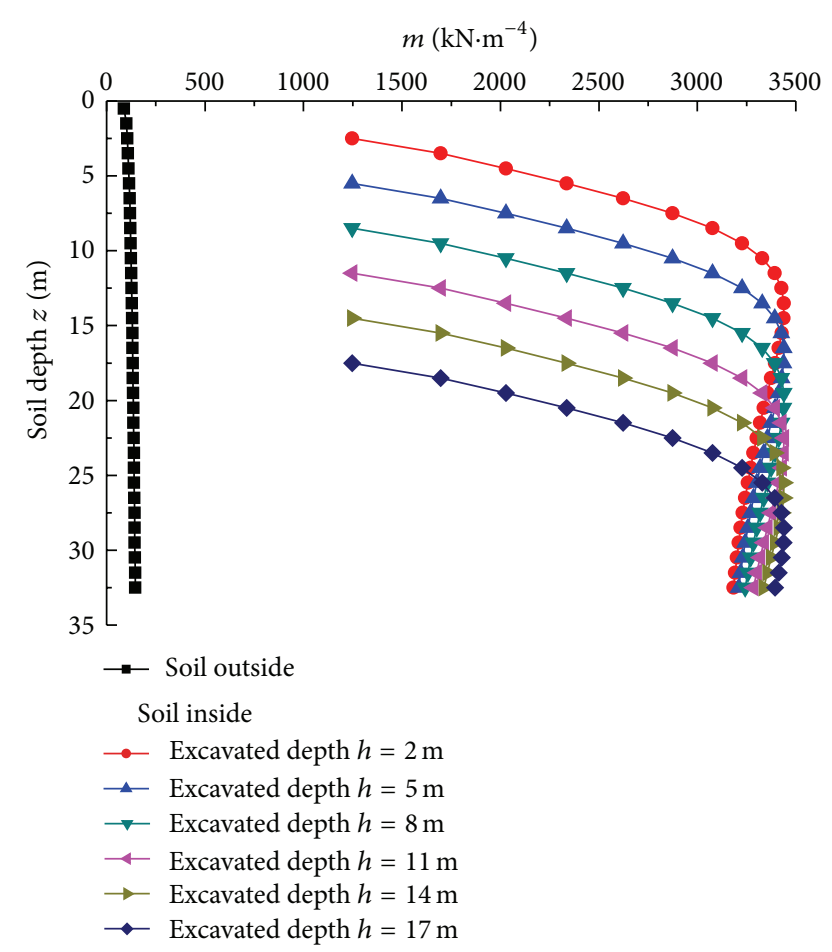

FIGURE 35: Horizontal foundation stiffness coefficients for each of six excavation steps.

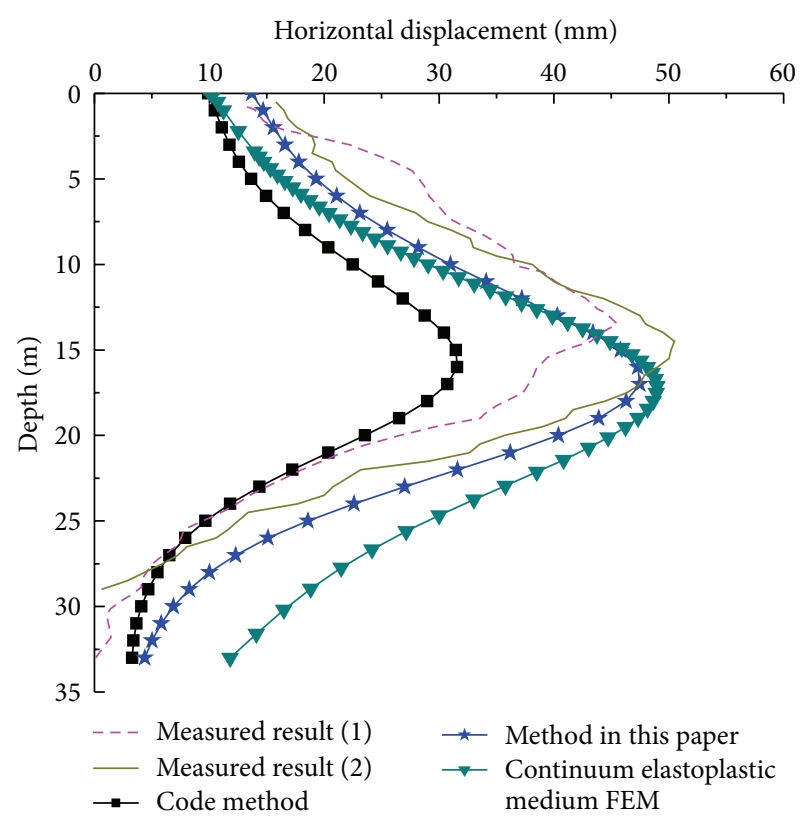

FIGURE 36: Deformation in retaining structures.

exhibits the smallest influence. When the soil depth is half of the foundation pit width, the proportional coefficient $m$ approaches a constant value. In general, the value of $m$ of the soil inside the pit is significantly larger than the value of $m$ of the soil outside the pit.

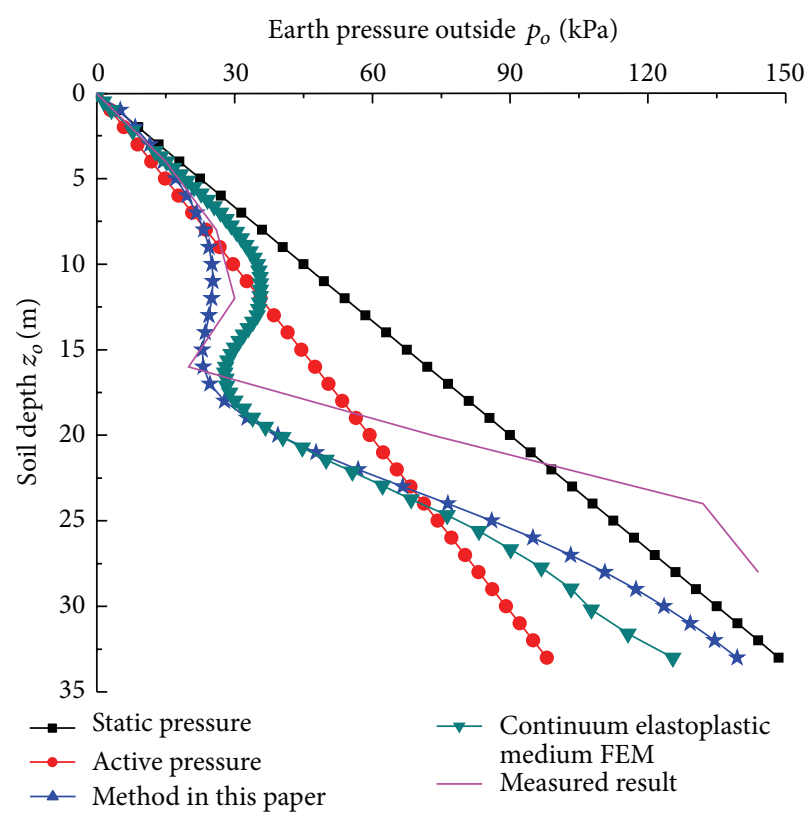

FIGURE 37: Earth pressure outside the pit.

(3) When the load level $q$ increases, the secant modulus of the soil and the value of the coefficient $\alpha$ decrease. When the strength parameter $b$ increases, the soil secant modulus and the value of the coefficient $\alpha$ decrease.

(4) Taking the influence of seepage into consideration, the horizontal foundation stiffness coefficient $K_{i}$ of the soil inside the pit can be expressed as $\alpha \cdot \lambda$. $\left(\gamma_{s}^{\prime}-\gamma_{w} \cdot \bar{i}_{i}\right) \cdot z_{i}$, and the horizontal foundation stiffness coefficient $K_{o}$ of the soil outside the pit can be expressed as $\alpha \cdot \lambda \cdot\left(\gamma_{s}^{\prime}+\gamma_{w} \cdot \bar{i}_{o}\right) \cdot z_{o}$. Seepage will cause the value of the horizontal foundation stiffness coefficient $K_{i}$ of the soil inside the pit to decrease and the coefficient $K_{o}$ of the soil outside the pit to increase.

(5) The results obtained for the example analysis and case study presented indicate that the model and method proposed in this paper yield results similar to measured results and similar to results obtained using a continuum elastic-plastic-medium finite element model. The good agreement among the three types of results indicates that the method and model proposed in this paper are capable of accurately calculating the forces and deformations of retaining structures in an ultradeep foundation pit.

\section{Acknowledgment}

The author would like to acknowledge the financial support from the National Natural Science Foundation of China (NSFC Grant no. 51108417). 


\section{References}

[1] P. R. Ministry of Metallurgical Industry China, "YB9258-97 Code for technique of building foundation pit engineering," Beijing, China, Metallurgical Industry Press, 1997.

[2] P. R. Ministry of Construction China, "JGJ120-99 Technical specification for retaining and protection of building foundation excavations," Beijing China, China Building Industry Press, 1999.

[3] R.-Q. Xu, "Methods of earth pressure calculation for excavation," Journal of Zhejiang University, vol. 34, no. 4, pp. 370-375, 2000.

[4] Y.-K. Chen, R.-Q. Xu, X.-J. Yang, and X.-N. Gong, "A new method calculating earth pressure on flexible structures for excavation work," Industrial Construction, vol. 31, no. 3, pp. 14, 2001.

[5] J.-P. Zhao, M. Guo-xiong, and J.-M. Zai, "Earth pressure model considering settlement and time effect," Journal of Yancheng Institute of Technology, vol. 4, pp. 55-63, 2003.

[6] L. Bei and X.-H. Zhao, "A nonlinear earth pressure method for deep excavation considering deformation of retaining wall," Rock and Soil Mechanics, vol. 25, no. 2, pp. 453-458, 2004.

[7] P. V. Lade and J. M. Duncan, "Stress-path dependent behavior of cohesionless soil," ASCE, Journal of the Geotechnical Engineering Division, vol. 102, no. 1, pp. 51-68, 1976.

[8] J. Yuan, X.-W. Liu, and D.-Q. Yi, "Determination of $m$ coefficient during excavation," Industrial Construction, vol. 30, no. 9, pp. 46-51, 2000.

[9] G. B. Liu and X.-Y. Hou, "Unloading modulus of the shanghai soft clay," Chinese Jounal of Geotechnical Engineering, vol. 18, no. 6, pp. 18-23, 1996.

[10] X.-Y. Liu, "Experimental and numerical simulation of excavation process and microstructure study," Tianjin, China, Tianjin Universiy, 2003.

[11] N. W. W. Charles and S. Qun, "Changes of stress path caused by stress relief during excavations," China Civil Engineering Journal, vol. 32, no. 6, pp. 53-58, 1999.

[12] Construction department of zhejiang province, "DB33/T10082000 Code for technique of building foundation excavation engineering," Zhejiang, China, 2000.

[13] Shanghai Uran Construction Committee, "DBJ-61-97 Code for design of excavation engineering," Shanghai, China, 1997.

[14] G.-M. Chen, J.-D. Zhong, and Z.-Q. Tang, "The discussion on calculation of soil lateral subgrade coefficient with flat dialatometer test," Shanghai Geology, vol. 2, pp. 40-42, 2002.

[15] Z.H. U. Bi-tang, "Limiting force profile and response of laterally loaded piles," Shanghai, China, Tongji University, 2005.

[16] M. T. Davission and H. L. Gill, "Laterally loaded piles in a layered soil system," ASCE Journal of the Soil Mechanics and Foundations Engineering, vol. 89, no. 3, pp. 63-94, 1963.

[17] H. G. Poulous and E. H. Davis, Elastic Solutions for Soil and Rock Mechanics, John Wiley \& Sons, New York, NY, USA, 1974.

[18] China Architecture \& Building Press, "Foundation and Basis," 1991.

[19] Q. Hu, "Study on design method and water-soil-retaining structure interaction of ultra-deep foundation pit," Hangzhou, China, Zhejiang University, 2008.

[20] J.-D. Wei, " Sudy on the earth pressure of silty soil and the behaviour of retaining structures for pit excavation," Hangzhou, China, Zhejiang University, 2006.
[21] J. M. Duncan and C. Y. Chang, "Nonlinear analysis of stress and strain in soils," ASCE Journal of the Soil Mechanics and Foundations Division, vol. 96, no. 5, pp. 1629-1653, 1970. 


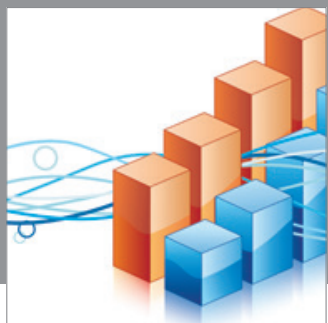

Advances in

Operations Research

mansans

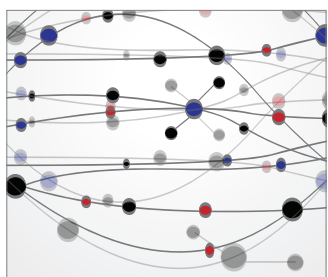

The Scientific World Journal
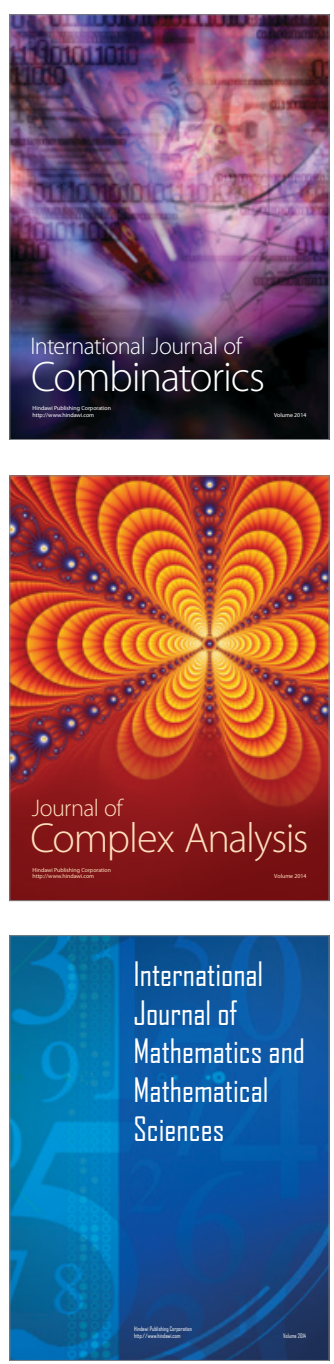
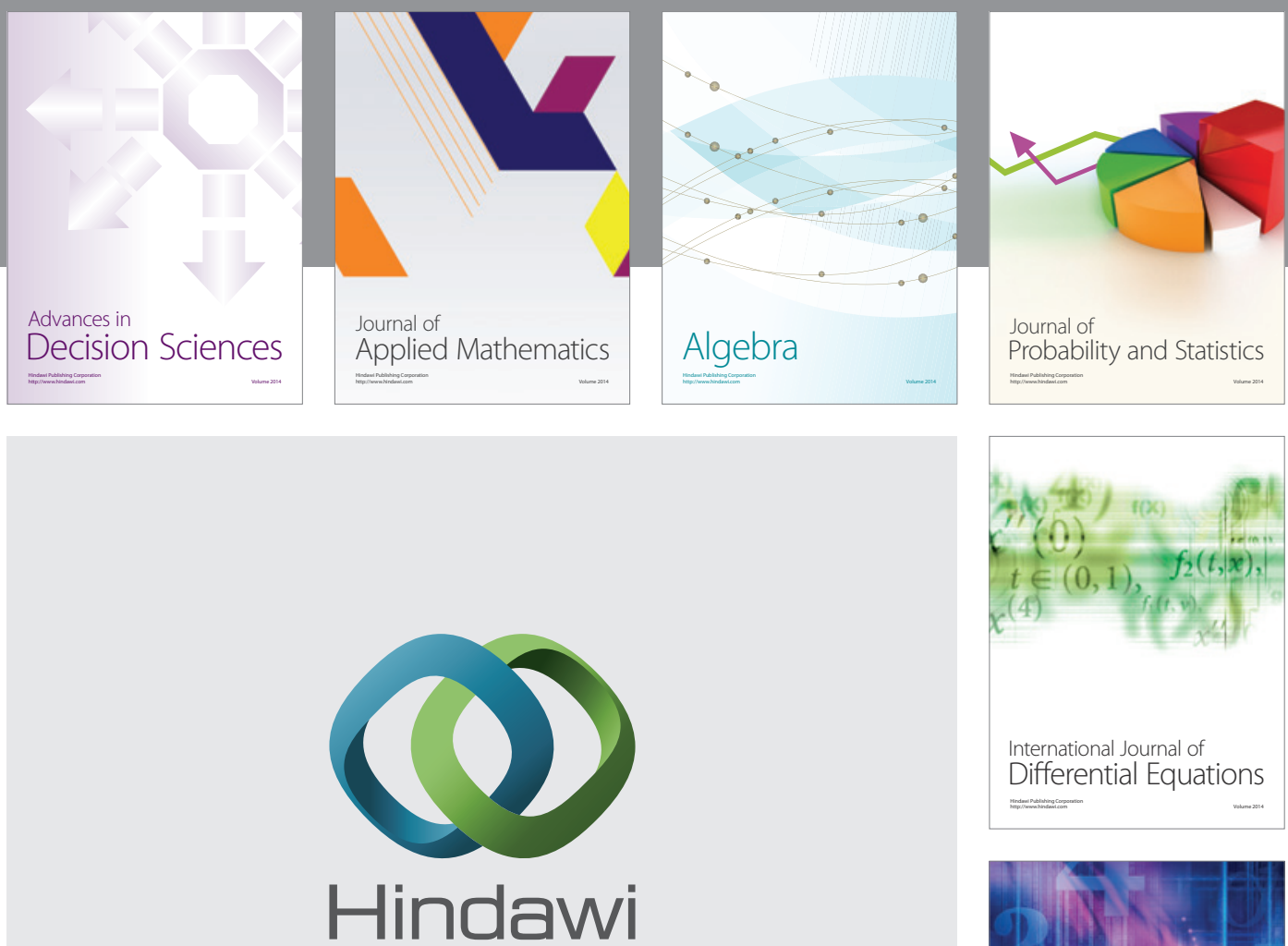

Submit your manuscripts at http://www.hindawi.com
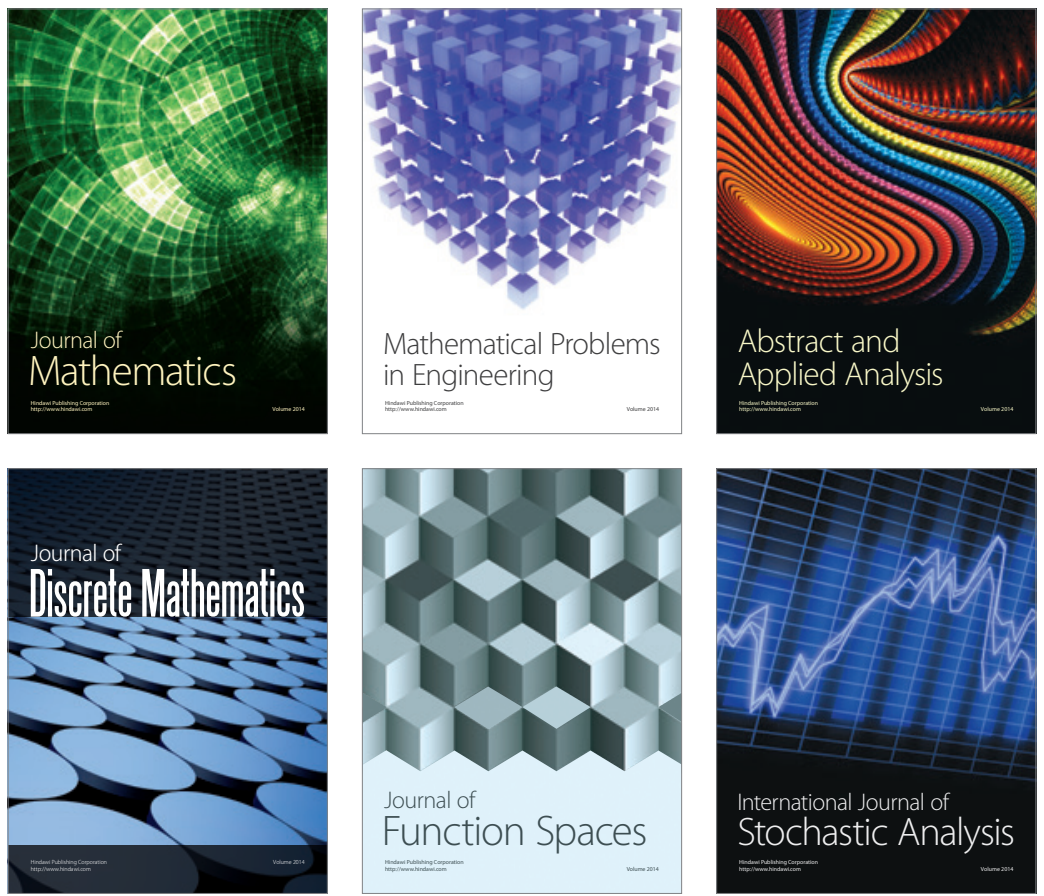

Journal of

Function Spaces

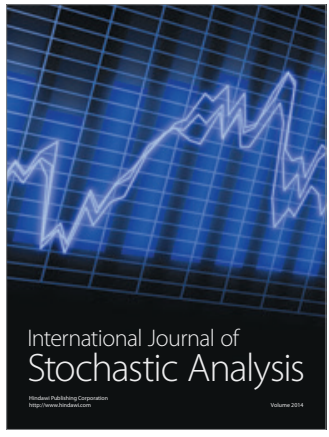

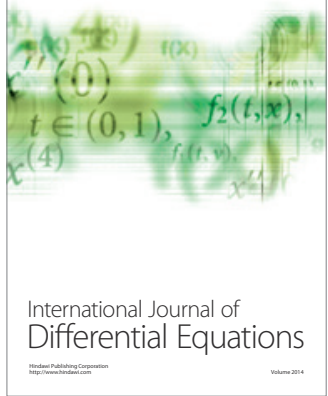
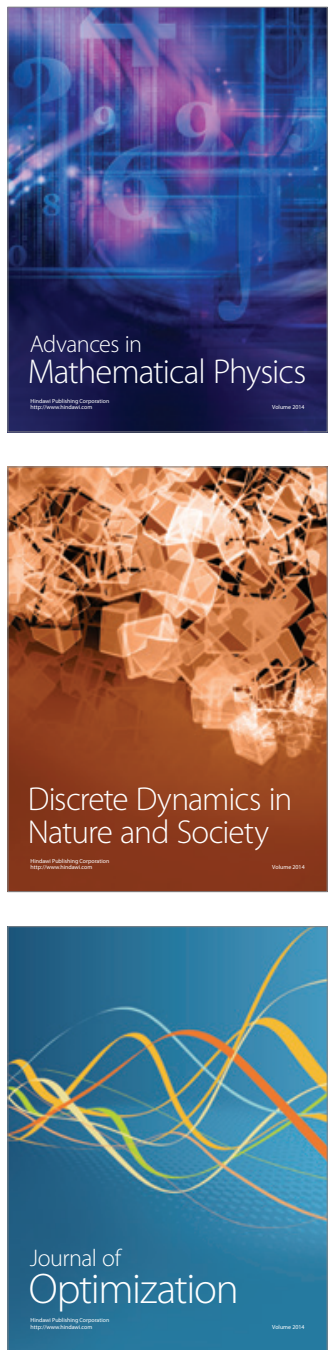